\title{
Orbifold family unification using vectorlike representation on six dimensions
}

\author{
Yuhei Goto ${ }^{1, *}$ and Yoshiharu Kawamura ${ }^{2, \dagger}$ \\ ${ }^{1}$ Research and Education Center for Natural Science, Keio University, Yokohama 223-8522, Japan \\ ${ }^{2}$ Department of Physics, Shinshu University, Matsumoto 390-8621, Japan
}

(Received 24 January 2018; published 27 August 2018)

\begin{abstract}
In orbifold family unification on the basis of $S U(N)$ gauge theory on the six-dimensional space-time $M^{4} \times T^{2} / Z_{m}(m=2,3,4,6)$, enormous numbers of models with three families of the standard model matter multiplets are derived from a massless Dirac fermion in a vectorlike representation $[N, 3]+[N, N-3]$ of $S U(N)(N=8,9)$. They contain models with three or more than three neutrino singlets and without any non-Abelian continuous flavor gauge symmetries. The relationship between flavor numbers from a fermion in $[N, N-k]$ and those from a fermion in $[N, k]$ are studied from the viewpoint of charge conjugation.
\end{abstract}

DOI: 10.1103/PhysRevD.98.035039

\section{INTRODUCTION}

One of the most intriguing riddles in particle physics is the origin of family replication in standard model (SM) matter multiplets. Various investigations have been performed, using models on the four-dimensional Minkowski space-time $M^{4}$ [1-7], but, in most cases, we encounter difficulties relating to the chiralness of fermions. Concretely, chiral fermions do not, in general, come from a fermion in an anomaly-free representation of a large gauge group, e.g., $\mathbf{2}^{n-1}$ for $S O(2 n)(n \geq 6)$, or a vectorlike (nonchiral) set of representations, e.g., $N+\bar{N}$ for $S U(N)$, as an extension of grand unified theories (GUTs). In most cases, particles with opposite quantum numbers under the SM gauge group $S U(3)_{C} \times S U(2)_{L} \times U(1)_{Y}$, called mirror particles, appear and the survival hypothesis is adopted to get rid of them from the low-energy spectrum. Then, the SM family members can also disappear. Here, the survival hypothesis is stated such that if a symmetry is broken down into a smaller one at a scale $M_{S}$, then any fermion mass terms invariant under the smaller group induce fermion masses of $O\left(M_{S}\right)$ and such heavy fermions disappear from the low-energy spectrum $[3,8] .{ }^{1}$

\footnotetext{
*y-goto@keio.jp

haru@azusa.shinshu-u.ac.jp

${ }^{1}$ There is a possibility that extra particles are confined at a high-energy scale by some strong dynamics $[2,6]$.

Published by the American Physical Society under the terms of the Creative Commons Attribution 4.0 International license. Further distribution of this work must maintain attribution to the author(s) and the published article's title, journal citation, and DOI. Funded by SCOAP ${ }^{3}$.
}

The above difficulty can be overcome by extending the structure of space-time. That is, extra particles, including mirror ones, can be eliminated using an orbifold breaking mechanism, as originally proposed in superstring theory [9-11]. Hence, a candidate realizing the family unification is an extension of GUTs defined on a higher-dimensional space-time including an orbifold. ${ }^{2}$ These studies have been carried out intensively [14-25], and three replicas of matter multiplets are derived from characteristics of extra dimensions. For instance, three replication $S U(5)$ multiplets have been derived from a single bulk fermion in the rank $k$ totally antisymmetric tensor representation $[N, k](N \geq 9)$ of $S U(N)$ on $M^{4} \times S^{1} / Z_{2}$ [20]. Enormous numbers of models with three families of the SM matter multiplets have been obtained from a single massless Dirac fermion in $[N, k]$ $(N \geq 9)$ of $S U(N)$ on $M^{4} \times T^{2} / Z_{m}(m=2,3,4)$ [23]. The relationship between the flavor numbers of chiral fermions and the Wilson line phases has been studied in these models [26]. Using models originated from $S U(9)$ gauge theory on $M^{4} \times T^{2} / Z_{2}$, their reality has been examined from the structure of the Yukawa interactions [27].

In Ref. [23], we find that the number of neutrino singlets is less than 3, the smallest gauge group is $S U(9)$, and most models contain an extra non-Abelian continuous gauge group relating to a flavor symmetry, under the precondition that three SM families are derived from a massless Dirac fermion in a chiral representation $[N, k]$ of $S U(N)$. Then, we need extra neutrino singlets to produce massive neutrinos and extra scalar fields to break extra gauge

\footnotetext{
${ }^{2}$ Five-dimensional supersymmetric GUTs on $M^{4} \times S^{1} / Z_{2}$ possess the attractive feature that the triplet-doublet splitting of Higgs multiplets is elegantly realized $[12,13]$.
} 
symmetries. By changing the precondition such that three SM families are derived from a massless Dirac fermion in a vectorlike representation $[N, k]+[N, N-k]$ of $S U(N)$, there is a possibility that some models possess features such that the number of neutrino singlets is 3 or more than 3 , the smallest gauge group is less than $S U(9)$, and all extra gauge symmetries are Abelian. Furthermore, extra gauge symmetries could be broken down by the vacuum expectation values of superpartners of neutrino singlets.

In this paper, we study the possibility of family unification on the basis of $S U(8)$ and $S U(9)$ gauge theory on $M^{4} \times T^{2} / Z_{m}$, using the method in Refs. [20,23]. We investigate whether or not three families of the SM matter multiplets are derived from a single massless Dirac fermion in a vectorlike representation $[8, k]+[8,8-k]$ or $[9, k]+$ $[9,9-k]$, through the orbifold breaking mechanism. We clarify the relationship between flavor numbers from a fermion in $[N, N-k]$ and those from a fermion in $[N, k]$ from the viewpoint of charge conjugation.

The contents of this paper are as follows. In Sec. II, we provide general arguments on the orbifold breaking based on the two-dimensional orbifold $T^{2} / Z_{m}$. In Sec. III, we give formulas for numbers of the SM matter multiplets. In Sec. IV, we study the possibility of the family unification in six-dimensional $S U(8)$ and $S U(9)$ gauge theories containing a massless Dirac fermion in a vectorlike representation. Section V is devoted to conclusions and discussions.

\section{II. $Z_{m}$ ORBIFOLD BREAKING, FERMIONS, AND DECOMPOSITION OF FIELD}

We explain the orbifold $T^{2} / Z_{m}(m=2,3,4,6)$, a sixdimensional fermion and a decomposition of field in $[N, k]$.

\section{A. $Z_{m}$ orbifold breaking}

On a two-dimensional lattice $T^{2}$, the points $z+e_{1}$ and $z+e_{2}$ are identified with the point $z$, where $e_{1}$ and $e_{2}$ are basis vectors and $z$ takes a complex value. The orbifold $T^{2} / Z_{m}$ is obtained by dividing $T^{2}$ by the $Z_{m}$ transformation $z \rightarrow \rho z$, where $\rho$ is the $m$ th root of unity $\left(\rho^{m}=1\right)$. Then, $z$ is identified with $\rho z$, or $z$ is identified with $\rho^{k} z+a e_{1}+b e_{2}$, where $k, a$, and $b$ are integers. For more details, see Appendix A.

We explain the $Z_{m}$ transformation properties of a six-dimensional scalar field $\Phi(x, z, \bar{z})$, using $T^{2} / Z_{3}$, whose basis vectors are given by $e_{1}=1$ and $e_{2}=i$. The extension of other fields (fermions and gauge bosons) and other orbifolds is straightforward. From the requirement that the Lagrangian density $\mathcal{L}$ should be invariant under the $Z_{3}$ transformations $s_{0}: z \rightarrow \omega z$ and $s_{1}: z \rightarrow \omega z+1$ $\left(\omega=e^{2 \pi i / 3}\right)$ or it should be a single-valued function,

$$
\begin{aligned}
\mathcal{L}(\Phi(x, \omega z, \bar{\omega} \bar{z})) & =\mathcal{L}(\Phi(x, z, \bar{z})), \\
\mathcal{L}(\Phi(x, \omega z+1, \bar{\omega} \bar{z}+1)) & =\mathcal{L}(\Phi(x, z, \bar{z})),
\end{aligned}
$$

the boundary conditions of fields on $T^{2} / Z_{3}$ are determined up to some overall $Z_{3}$ factors, which we refer to as intrinsic $Z_{3}$ elements of fields and denote as $\eta_{a \Phi}$ corresponding to the $Z_{3}$ transformations $s_{a}(a=0,1)$. When $\Phi$ is a multiplet of some transformation group $G$ concerning some internal symmetries (including gauge symmetries), $\mathcal{L}$ should be invariant under the transformation $\Phi(x, z, \bar{z}) \rightarrow \Phi^{\prime}(x, z, \bar{z})=T_{\Phi} \Phi(x, z, \bar{z})$, such that

$$
\mathcal{L}\left(T_{\Phi} \Phi(x, z, \bar{z})\right)=\mathcal{L}(\Phi(x, z, \bar{z}))
$$

where $T_{\Phi}$ is a representation matrix of $G$ on $\Phi$. For instance, if a theory has $S U(N)$ gauge symmetry, $\mathcal{L}$ is, in general, invariant under a (global) $U(N)$ transformation, i.e., $G=U(N)$. From (2.1) and (2.2), the following boundary conditions on $\Phi$ are allowed:

$$
\begin{aligned}
\Phi(x, \omega z, \bar{\omega} \bar{z}) & =T_{\Phi}\left[U_{0}, \eta_{0 \Phi}\right] \Phi(x, z, \bar{z}), \\
\Phi(x, \omega z+1, \bar{\omega} \bar{z}+1) & =T_{\Phi}\left[U_{1}, \eta_{1 \Phi}\right] \Phi(x, z, \bar{z}),
\end{aligned}
$$

where $T_{\Phi}\left[U_{0}, \eta_{0 \Phi}\right]$ and $T_{\Phi}\left[U_{1}, \eta_{1 \Phi}\right]$ represent appropriate representation matrices, which are elements of $G$ on $\Phi$. The $T_{\Phi}\left[U_{a}, \eta_{a \Phi}\right]$ are factorized into $T_{\Phi}\left[U_{a}, \eta_{a \Phi}\right]=\eta_{a \Phi} \tilde{T}_{\Phi}\left[U_{a}\right]$ $(a=0,1)$, using representation matrices $U_{a}$ for the fundamental representations of $G$ and the intrinsic $Z_{3}$ elements $\eta_{a \Phi}$ [see (2.13)], and some relations can appear among the intrinsic $Z_{3}$ elements [see (2.6) or (B20)]. Arbitrary $U_{0}$ and $U_{1}$ can be diagonalized by using a global unitary transformation and a local gauge transformation or each equivalence class of boundary conditions contains diagonal representatives [28]. Hence we use diagonal ones later.

We list basis vectors and the transformations relating to identifications of points on $T^{2} / Z_{m}$, and we denote its representation matrices for the fundamental representation as $U_{a}\left(a=0,1,2\right.$ for $T^{2} / Z_{2} ; a=0,1$ for $T^{2} / Z_{3}$ and $T^{2} / Z_{4}$; and $a=0$ for $\left.T^{2} / Z_{6}\right)$ in Table I $[29,30]$. Note that there is a choice in transformations independently of each other.

Components of $\Phi$ possess discrete charges associated with eigenvalues of $T_{\Phi}\left[U_{a}, \eta_{a \Phi}\right]$. When the eigenvalues are given as $e^{2 \pi i l / m}(l=0,1, \ldots, m-1)$, the discrete charges are assigned as numbers $l / m$. We refer to $e^{2 \pi i l / m}$ as $Z_{m}$ elements. In the absence of contributions from the Wilson line phases, the massless six-dimensional fields whose $Z_{m}$ elements for all $a$ are equal to 1 contain zero modes, but those including a $Z_{m}$ element different from 1 do not contain zero modes. ${ }^{3}$ Here, zero modes mean four-dimensional massless fields. If the size of extra dimensions is small enough, massive modes called Kaluza-Klein modes

\footnotetext{
${ }^{3}$ In the presence of nonvanishing Wilson line phases, gauge symmetries and particle spectra are rearranged via the Hosotani mechanism [31-34].
} 
TABLE I. The characters of $T^{2} / Z_{m}$.

\begin{tabular}{lccc}
\hline \hline$T^{2} / Z_{m}$ & Basis vectors & Transformations & Representation matrices \\
\hline$T^{2} / Z_{2}$ & $1, i$ & $z \rightarrow-z, z \rightarrow 1-z, z \rightarrow i-z$ & $U_{0}, U_{1}, U_{2}$ \\
$T^{2} / Z_{3}$ & $1, e^{2 \pi i / 3}$ & $z \rightarrow e^{2 \pi i / 3} z, z \rightarrow e^{2 \pi i / 3} z+1$ & $U_{0}, U_{1}$ \\
$T^{2} / Z_{4}$ & $1, i$ & $z \rightarrow i z, z \rightarrow i z+1$ & $U_{0}, U_{1}$ \\
$T^{2} / Z_{6}$ & $1,(-3+i \sqrt{3}) / 2$ & $z \rightarrow e^{\pi i / 3} z$ & $U_{0}$ \\
\hline \hline
\end{tabular}

do not appear in low-energy theories. Unless all components of a nonsinglet field have a common $Z_{m}$ charge, a symmetry reduction occurs upon compactification. This type of symmetry breaking mechanism is called an "orbifold breaking mechanism."

\section{B. Fermions}

We explain fermions in six dimensions. For more details, see Appendix B. A massless Weyl fermion in six dimensions is regarded as a Dirac fermion or a pair of Weyl fermions with opposite chiralities in four dimensions. The six-dimensional Dirac fermion consists of two six-dimensional Weyl fermions such that

$$
\begin{aligned}
& \Psi_{+}=\frac{1+\Gamma_{7}}{2} \Psi=\left(\begin{array}{c}
\psi_{+L} \\
\psi_{+R}
\end{array}\right), \\
& \Psi_{-}=\frac{1-\Gamma_{7}}{2} \Psi=\left(\begin{array}{c}
\psi_{-R} \\
\psi_{-L}
\end{array}\right),
\end{aligned}
$$

where $\Psi_{+}$and $\Psi_{-}$are fermions with positive and negative chirality, respectively, and $\Gamma_{7}$ is the chirality operator in six dimensions. Here and hereafter, the subscript \pm and $L(R)$ stand for the chiralities in six and four dimensions, respectively. The charge conjugation of a six-dimensional Dirac fermion $\Psi$ is defined as

$$
\Psi^{c} \equiv B \Psi^{*}, \quad B^{-1} \Gamma^{M} B=-\left(\Gamma^{M}\right)^{*},
$$

where $\Gamma^{M}(M=0,1,2,3,5,6)$ are six-dimensional gamma matrices, $B=-i \Gamma_{7} \Gamma^{2} \Gamma^{5}$ up to a phase factor, and the asterisk $*$ means the complex conjugation. ${ }^{5}$ Note that the chirality in six dimensions does not flip under the charge conjugation, as shown in (B12) and (B13).

From the $Z_{m}$ invariance of the kinetic term and the transformation property of the covariant derivatives $D_{z} \rightarrow \bar{\rho} D_{z}$ and $D_{\bar{z}} \rightarrow \rho D_{\bar{z}}$ with $\bar{\rho}\left(=\rho^{*}\right)=e^{-2 \pi i / m}$ and $\rho=e^{2 \pi i / m}$, we have the relations

\footnotetext{
${ }^{4}$ The $Z_{2}$ orbifolding was used in superstring theory [35] and heterotic M-theory [36,37]. In field theoretical models, it was applied to the reduction of global supersymmetry (SUSY) $[38,39]$, which is an orbifold version of the Scherk-Schwarz mechanism $[40,41]$, and then to the reduction of gauge symmetry [42].

${ }^{5}$ In this paper, the complex conjugation is also represented by the overlined one.
}

$$
\eta_{a+R}=\rho \eta_{a+L}, \quad \eta_{a-R}=\bar{\rho} \eta_{a-L},
$$

where $z \equiv x^{5}+i x^{6}$ and $\bar{z} \equiv x^{5}-i x^{6}$, and $\eta_{a \pm L(R)}$ are the intrinsic $Z_{m}$ elements of $\psi_{ \pm L(R)}$. For the derivation of (2.6), see (B14)-(B20).

Chiral gauge theories including Weyl fermions in evendimensional space-time become, in general, anomalous in the presence of gauge anomalies, gravitational anomalies, mixed anomalies, and/or a global anomaly $[43,44]$. Here we consider a nonsupersymmetric model for simplicity. In $S U(N)$ gauge theories in six dimensions, the global anomaly is absent because of $\pi_{6}(S U(N))=0$ for $N \geq 4$. Here, $\pi_{6}(S U(N))$ is the sixth homotopy group of $S U(N)$. Other anomalies must be canceled out by the contributions from several fermions. For instance, they are canceled out by the contributions from fermions with different chiralities such as $\left(\Psi_{+}^{r}, \Psi_{-}^{r}\right)$, where $\boldsymbol{r}$ stands for the $r$-dimensional representation of $S U(N)$. Each pair in $\left(\Psi_{+}^{r}, \Psi_{-}^{\bar{r}}\right) ;\left(\Psi_{+}^{\bar{r}_{+}}, \Psi_{-}^{r}\right)$; and $\left(\Psi_{+}^{\bar{r}}, \Psi_{-}^{\bar{r}}\right)$ does not contribute to the anomalies, where $\bar{r}$ stands for the complex conjugate representation of $\boldsymbol{r}$. It is understood that the cancellation in six dimensions occurs from the fact that the gauge anomaly is proportional to a group-theoretical factor such as

$$
\sum_{\Psi_{+}} \operatorname{Str}\left(T^{a_{1}} T^{a_{2}} T^{a_{3}} T^{a_{4}}\right)-\sum_{\Psi_{-}} \operatorname{Str}\left(T^{a_{1}} T^{a_{2}} T^{a_{3}} T^{a_{4}}\right),
$$

where Str stands for the trace over the symmetrized product of the gauge group generators $T^{a_{i}}$, and this trace is invariant under the exchange between $T^{a_{i}}$ and $-\left(T^{a_{i}}\right)^{*}$, corresponding to the exchange between a fermion in $\boldsymbol{r}$ and one in $\overline{\boldsymbol{r}}$. The gravitational anomaly is canceled out, if the following condition is fulfilled:

$$
N_{+}=N_{-},
$$

where $N_{ \pm}$are the numbers (including degrees of freedom) of $\Psi_{ \pm}$.

\section{Decomposition of representation}

With suitable diagonal representation matrices $U_{a}$, the $S U(N)$ gauge group is broken down into its subgroup such that

$S U(N) \rightarrow S U\left(p_{1}\right) \times S U\left(p_{2}\right) \times \cdots \times S U\left(p_{n}\right) \times U(1)^{n-n^{\prime}-1}$, 
where $N=p_{1}+p_{2}+\cdots+p_{n}$. Here and hereafter, $S U(1)$ unconventionally stands for $U(1), S U(0)$ means nothing, and $n^{\prime}$ is a sum of the number of $S U(0)$. A concrete form of $U_{a}$ will be given in the next section.

After the breakdown of $S U(N)$, the rank $k$ totally antisymmetric tensor representation $[N, k]$, whose dimension is ${ }_{N} C_{k}$, is decomposed into a sum of multiplets of the subgroup $S U\left(p_{1}\right) \times S U\left(p_{2}\right) \times \cdots \times S U\left(p_{n}\right)$ as

$[N, k]=\sum_{l_{1}=0}^{k} \sum_{l_{2}=0}^{k-l_{1}} \ldots \sum_{l_{n-1}=0}^{k-l_{1}-\cdots-l_{n-2}}\left({ }_{p_{1}} C_{l_{1}},{ }_{p_{2}} C_{l_{2}}, \ldots,{ }_{p_{n}} C_{l_{n}}\right)$,

where $l_{n}=k-l_{1}-\cdots-l_{n-1}$ and our notation is that ${ }_{n} C_{l}=0$ for $l>n$ and $l<0$. Here and hereafter, we use ${ }_{n} C_{l}$ instead of $[n, l]$ in many cases. We sometimes use the ordinary notation for representations too, e.g., $N$ and $\bar{N}$ in place of ${ }_{N} C_{1}$ and ${ }_{N} C_{N-1}$.

The $[N, k]$ is constructed by the antisymmetrization of the $k$-ple product of the fundamental representation $N=[N, 1]:$

$$
[N, k]=(\underbrace{N \times \ldots \times N}_{k})_{A},
$$

where a tiny subscript $A$ means the antisymmetrization. For Weyl fermions $\Psi_{ \pm}$in $[N, k]$, the boundary conditions are given by

$$
\Psi_{ \pm}(x, \rho z, \bar{\rho} \bar{z})=T_{\Psi \pm}\left[U_{a}, \eta_{a \pm}^{(k)}\right] \Psi_{ \pm}(x, z, \bar{z}),
$$

where $T_{\Psi_{ \pm}}\left[U_{a}, \eta_{a \pm}^{(k)}\right]$ stand for appropriate representation matrices, which are elements of $U(N)$ on $\Psi_{ \pm}, U_{a}$ are the representation matrices for the fundamental representation, and $\eta_{a \pm}^{(k)}$ are the intrinsic $Z_{m}$ elements of $\Psi_{ \pm}$in $[N, k]$. We omit the subscripts $L$ and $R$ on $\eta_{a \pm}^{(k)}$, for simplicity. Note that there are relations such as (2.6) between $\eta_{a \pm L}^{(k)}$ and $\eta_{a \pm R}^{(k)}$. Using (2.11) and (2.12), the $Z_{m}$ transformation property of $[N, k]$ can be expressed by

$(\boldsymbol{N} \times \ldots \times \boldsymbol{N})_{A} \rightarrow \eta_{a \pm}^{(k)}\left(\left(U_{a} \boldsymbol{N}\right) \times \ldots \times\left(U_{a} \boldsymbol{N}\right)\right)_{A}$.

By definition, $\eta_{a \pm}^{(k)}$ take values of $Z_{m}$ elements, i.e., $e^{2 \pi i l / m}$ $(l=0,1, \ldots, m-1)$. Note that $\eta_{a+}^{(k)}$ are not necessarily the same as $\eta_{a-}^{(k)}$, and the chiral symmetry is still respected.

In the same way, the $[N, N-k]$ is constructed by the antisymmetrization of the $(N-k)$-ple product of $N$,

$$
[N, N-k]=(\underbrace{N \times \ldots \times N}_{N-k})_{A},
$$

or it is also constructed by the antisymmetrization of the $k$-ple product of the complex conjugate representation $\bar{N}$ :

$$
[N, N-k]=\overline{[N, k]}=(\underbrace{\bar{N} \times \ldots \times \bar{N}}_{k})_{A} .
$$

Using (2.15), the $Z_{m}$ transformation property is given by

$$
(\overline{\boldsymbol{N}} \times \ldots \times \overline{\boldsymbol{N}})_{A} \rightarrow \tilde{\eta}_{a \pm}^{(k)}\left(\left(U_{a}^{*} \overline{\boldsymbol{N}}\right) \times \ldots \times\left(U_{a}^{*} \overline{\boldsymbol{N}}\right)\right)_{A},
$$

where $U_{a}^{*}$ are the complex conjugations of $U_{a}$, and $\tilde{\eta}_{a \pm}^{(k)}$ are the intrinsic $Z_{m}$ elements of $\Psi_{ \pm}$in $\overline{[N, k]}$. If the field in $\overline{[N, k]}$ is obtained by the charge conjugation of that in $[N, k]$, we have relations $\tilde{\eta}_{a \pm}^{(k)}=\overline{\eta_{a \pm}^{(k)}}$. Strictly speaking, in this case, the relations are written as $\tilde{\eta}_{a \pm R}^{(k)}=\overline{\eta_{a \pm L}^{(k)}}$ and $\tilde{\eta}_{a \pm L}^{(k)}=\overline{\eta_{a \pm R}^{(k)}}$, because the four-dimensional chirality changes under the charge conjugation. If a field in $[N, k]$ is independent of that in $\overline{[N, k]}$, there is no relation between $\eta_{a \pm}^{(k)}$ and $\tilde{\eta}_{a \pm}^{(k)}$.

\section{FORMULAS FOR NUMBERS OF SM SPECIES}

Let us investigate the family unification with the breaking pattern:

$$
\begin{aligned}
S U(N) \rightarrow & S U(3) \times S U(2) \times S U\left(p_{3}\right) \\
& \times \cdots \times S U\left(p_{n}\right) \times U(1)^{n-n^{\prime}-1},
\end{aligned}
$$

where $S U(3)$ and $S U(2)$ are identified with $S U(3)_{C}$ and $S U(2)_{L}$ in the SM gauge group. After the breakdown of $S U(N),[N, k]$ is decomposed into a sum of multiplets as

$$
\begin{aligned}
{[N, k]=} & \sum_{l_{1}=0}^{k} \sum_{l_{2}=0}^{k-l_{1}} \sum_{l_{3}=0}^{k-l_{1}-l_{2}} \ldots \\
& \times \sum_{l_{n-1}=0}^{k-l_{1}-\ldots-l_{n-2}}\left({ }_{3} C_{l_{1}},{ }_{2} C_{l_{2}},{ }_{p_{3}} C_{l_{3}}, \ldots,{ }_{p_{n}} C_{l_{n}}\right) .
\end{aligned}
$$

The flavor numbers of down-type antiquark singlets $\left(d_{R}\right)^{c}$, lepton doublets $l_{L}$, up-type antiquark singlets $\left(u_{R}\right)^{c}$, positron-type lepton singlets $\left(e_{R}\right)^{c}$, and quark doublets $q_{L}$ are denoted as $n_{\bar{d}}, n_{l}, n_{\bar{u}}, n_{\bar{e}}$, and $n_{q}$, respectively. Using the survival hypothesis and the equivalence on charge conjugation in four dimensions, we define the flavor number of each SM chiral fermion as

$$
\begin{aligned}
n_{\bar{d}} \equiv & \left(\sharp\left({ }_{3} C_{2},{ }_{2} C_{2}\right)_{L}-\sharp\left({ }_{3} C_{1},{ }_{2} C_{0}\right)_{L}\right) \\
& -\left(\sharp\left({ }_{3} C_{2},{ }_{2} C_{2}\right)_{R}-\sharp\left({ }_{3} C_{1},{ }_{2} C_{0}\right)_{R}\right), \\
n_{l} \equiv & \left(\sharp\left({ }_{3} C_{3},{ }_{2} C_{1}\right)_{L}-\sharp\left({ }_{3} C_{0},{ }_{2} C_{1}\right)_{L}\right) \\
& -\left(\sharp\left({ }_{3} C_{3},{ }_{2} C_{1}\right)_{R}-\sharp\left({ }_{3} C_{0},{ }_{2} C_{1}\right)_{R}\right),
\end{aligned}
$$




$$
\begin{aligned}
n_{\bar{u}} \equiv & \left(\sharp\left({ }_{3} C_{2},{ }_{2} C_{0}\right)_{L}-\sharp\left({ }_{3} C_{1},{ }_{2} C_{2}\right)_{L}\right) \\
& -\left(\sharp\left({ }_{3} C_{2},{ }_{2} C_{0}\right)_{R}-\sharp\left({ }_{3} C_{1},{ }_{2} C_{2}\right)_{R}\right), \\
n_{\bar{e}} \equiv & \left(\sharp\left({ }_{3} C_{0},{ }_{2} C_{2}\right)_{L}-\sharp\left({ }_{3} C_{3},{ }_{2} C_{0}\right)_{L}\right) \\
& -\left(\sharp\left({ }_{3} C_{0},{ }_{2} C_{2}\right)_{R}-\sharp\left({ }_{3} C_{3},{ }_{2} C_{0}\right)_{R}\right), \\
n_{q} \equiv & \left(\sharp\left({ }_{3} C_{1},{ }_{2} C_{1}\right)_{L}-\sharp\left({ }_{3} C_{2},{ }_{2} C_{1}\right)_{L}\right) \\
& -\left(\sharp\left({ }_{3} C_{1},{ }_{2} C_{1}\right)_{R}-\sharp\left({ }_{3} C_{2},{ }_{2} C_{1}\right)_{R}\right),
\end{aligned}
$$

where $\sharp$ represents the number of zero modes for each multiplet. The SM singlets are regarded as the right-handed neutrinos, which can obtain heavy Majorana masses among themselves as well as the Dirac masses with left-handed neutrinos. Some of them can be involved in the seesaw mechanism $[2,45,46]$. The total number of (heavy) neutrino singlets $\left(\nu_{R}\right)^{c}$ and/or $\nu_{R}$ is denoted by $n_{\bar{\nu}}$ and defined as

$$
\begin{aligned}
n_{\bar{\nu}} \equiv & \sharp\left({ }_{3} C_{0},{ }_{2} C_{0}\right)_{L}+\sharp\left({ }_{3} C_{3},{ }_{2} C_{2}\right)_{L}+\sharp\left({ }_{3} C_{0},{ }_{2} C_{0}\right)_{R} \\
& +\sharp\left({ }_{3} C_{3},{ }_{2} C_{2}\right)_{R} .
\end{aligned}
$$

From (3.2), the number of zero modes for each multiplet is given by the formulas
TABLE II. The projection operators $P_{m k \pm L(R)}$.

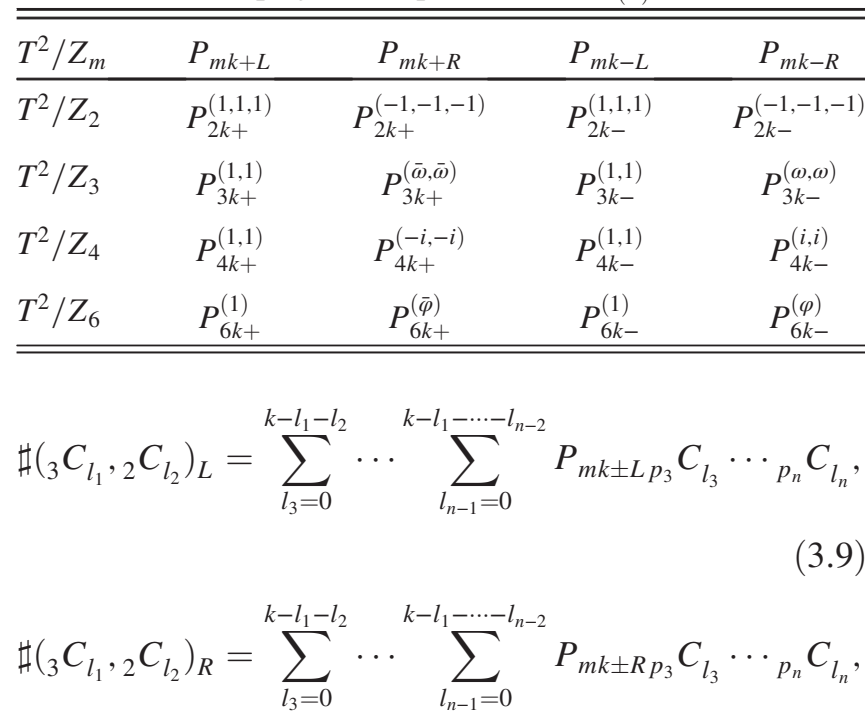

where the $P_{m k \pm L(R)}(m=2,3,4,6)$ are projection operators to pick out zero modes of $\psi_{ \pm L(R)}$ in $[N, k]$, and they are listed in Table II.

In Table II, $\varphi=e^{i \pi / 3}$ and $\bar{\varphi}=e^{-i \pi / 3}$, and each operator is defined by

$$
\begin{aligned}
& P_{2 k \pm}^{\left((-1)^{n_{0}},(-1)^{n_{1}},(-1)^{n_{2}}\right)} \equiv \frac{1}{8}\left\{1+(-1)^{n_{0}} \mathcal{P}_{0 \pm}^{(k)}\right\}\left\{1+(-1)^{n_{1}} \mathcal{P}_{1 \pm}^{(k)}\right\}\left\{1+(-1)^{n_{2}} \mathcal{P}_{2 \pm}^{(k)}\right\} \\
& P_{3 k \pm}^{\left(\omega^{n_{0}}, \omega^{n_{1}}\right)} \equiv \frac{1}{9}\left\{1+\bar{\omega}^{n_{0}} \mathcal{P}_{0 \pm}^{(k)}+\bar{\omega}^{2 n_{0}}\left(\mathcal{P}_{0 \pm}^{(k)}\right)^{2}\right\}\left\{1+\bar{\omega}^{n_{1}} \mathcal{P}_{1 \pm}^{(k)}+\bar{\omega}^{2 n_{1}}\left(\mathcal{P}_{1 \pm}^{(k)}\right)^{2}\right\}, \\
& P_{4 k \pm}^{\left(i^{n_{0}}, i^{n_{1}}\right)} \equiv \frac{1}{16}\left\{1+(-i)^{n_{0}} \mathcal{P}_{0 \pm}^{(k)}+(-i)^{2 n_{0}}\left(\mathcal{P}_{0 \pm}^{(k)}\right)^{2}+(-i)^{3 n_{0}}\left(\mathcal{P}_{0 \pm}^{(k)}\right)^{3}\right\} \\
& \times\left\{1+(-i)^{n_{1}} \mathcal{P}_{1 \pm}^{(k)}+(-i)^{2 n_{1}}\left(\mathcal{P}_{1 \pm}^{(k)}\right)^{2}+(-i)^{3 n_{1}}\left(\mathcal{P}_{1 \pm}^{(k)}\right)^{3}\right\}, \\
& P_{6 k \pm}^{\left(\varphi^{n_{0}}\right)} \equiv \frac{1}{6}\left\{1+\bar{\varphi}^{n_{0}} \mathcal{P}_{0 \pm}^{(k)}+\bar{\varphi}^{2 n_{0}}\left(\mathcal{P}_{0 \pm}^{(k)}\right)^{2}+\bar{\varphi}^{3 n_{0}}\left(\mathcal{P}_{0 \pm}^{(k)}\right)^{3}+\bar{\varphi}^{4 n_{0}}\left(\mathcal{P}_{0 \pm}^{(k)}\right)^{4}+\bar{\varphi}^{5 n_{0}}\left(\mathcal{P}_{0 \pm}^{(k)}\right)^{5}\right\}
\end{aligned}
$$

where $n_{0}, n_{1}$, and $n_{2}$ are integers, and $\mathcal{P}_{a \pm}^{(k)}$ are the $Z_{m}$ elements determined by $U_{a}$ and $\eta_{a \pm L(R)}^{(k)}$, as will be given below. For instance, $P_{3 k \pm}^{\left(\omega^{n_{0}}, \omega^{n_{1}}\right)}$ is a projection operator to pick out modes with $\mathcal{P}_{0 \pm}^{(k)}=\omega^{n_{0}}$ and $\mathcal{P}_{1 \pm}^{(k)}=\omega^{n_{1}}$ in $\Psi_{ \pm}$.

From (3.3)-(3.10), we obtain following formulas for the SM species and neutrino singlets derived from a pair of sixdimensional Weyl fermions $\left(\Psi_{+}, \Psi_{-}\right)$in $[N, k]$,

$$
\begin{aligned}
& \left.n_{\bar{d}}\right|_{[N, k]}=\sum_{ \pm} \sum_{\left(l_{1}, l_{2}\right)=(2,2),(1,0)} \sum_{l_{3}=0}^{k-l_{1}-l_{2}} \cdots \sum_{l_{n-1}=0}^{k-l_{1}-\cdots-l_{n-2}}(-1)^{l_{1}+l_{2}} P_{m k \pm p_{3}} C_{l_{3}} \cdots{ }_{p_{n}} C_{l_{n}}, \\
& \left.n_{l}\right|_{[N, k]}=\sum_{ \pm} \sum_{\left(l_{1}, l_{2}\right)=(3,1),(0,1)} \sum_{l_{3}=0}^{k-l_{1}-l_{2}} \cdots \sum_{l_{n-1}=0}^{k-l_{1}-\cdots-l_{n-2}}(-1)^{l_{1}+l_{2}} P_{m k \pm p_{3}} C_{l_{3}} \cdots{ }_{p_{n}} C_{l_{n}}, \\
& \left.n_{\bar{u}}\right|_{[N, k]}=\sum_{ \pm} \sum_{\left(l_{1}, l_{2}\right)=(2,0),(1,2)} \sum_{l_{3}=0}^{k-l_{1}-l_{2}} \cdots \sum_{l_{n-1}=0}^{k-l_{1}-\cdots-l_{n-2}}(-1)^{l_{1}+l_{2}} P_{m k \pm p_{3}} C_{l_{3}} \cdots{ }_{p_{n}} C_{l_{n}},
\end{aligned}
$$




$$
\begin{gathered}
\left.n_{\bar{e}}\right|_{[N, k]}=\sum_{ \pm} \sum_{\left(l_{1}, l_{2}\right)=(0,2),(3,0)} \sum_{l_{3}=0}^{k-l_{1}-l_{2}} \cdots \sum_{l_{n-1}=0}^{k-l_{1}-\cdots-l_{n-2}}(-1)^{l_{1}+l_{2}} P_{m k \pm p_{3}} C_{l_{3}} \cdots{ }_{p_{n}} C_{l_{n}}, \\
\left.n_{q}\right|_{[N, k]}=\sum_{ \pm} \sum_{\left(l_{1}, l_{2}\right)=(1,1),(2,1)} \sum_{l_{3}=0}^{k-l_{1}-l_{2}} \cdots \sum_{l_{n-1}=0}^{k-l_{1}-\cdots-l_{n-2}}(-1)^{l_{1}+l_{2}} P_{m k \pm p_{3}} C_{l_{3}} \cdots p_{n} C_{l_{n}}, \\
n_{\left.\bar{\nu}\right|_{[N, k]}}=\sum_{ \pm} \sum_{\left(l_{1}, l_{2}\right)=(0,0),(3,2)} \sum_{l_{3}=0}^{k-l_{1}-l_{2}} \sum_{l_{n-1}=0}^{k-l_{1}-\cdots-l_{n-2}} P_{m k \pm p_{3}}^{(\nu)} C_{l_{3}} \cdots_{p_{n}} C_{l_{n}},
\end{gathered}
$$

where $P_{m k \pm}$ and $P_{m k \pm}^{(\nu)}$ are defined by

$$
P_{m k \pm} \equiv P_{m k \pm L}-P_{m k \pm R}, \quad P_{m k \pm}^{(\nu)} \equiv P_{m k \pm L}+P_{m k \pm R},
$$

respectively. By the insertion of $(-1)^{l_{1}+l_{2}}$, we obtain $\sharp\left({ }_{3} C_{l_{1}},{ }_{2} C_{l_{2}}\right)_{L(R)}$ for $l_{1}+l_{2}=$ even integer and $-\sharp\left({ }_{3} C_{l_{1}},{ }_{2} C_{l_{2}}\right) L(R)$ for $l_{1}+l_{2}=$ odd integer. Although the above formulas (3.15)-(3.19) are derived with no consideration for the Wilson line phases, they still hold for the case with nonvanishing Wilson line phases relating to extra gauge symmetries, thanks to a hidden quantummechanical supersymmetry [26].

We explain how the $Z_{m}$ elements $\mathcal{P}_{a \pm}^{(k)}$ of multiplets in $\left({ }_{3} C_{l_{1}},{ }_{2} C_{l_{2}}, \ldots, p_{n} C_{l_{n}}\right)$ decomposed from $\Psi_{ \pm}$in $[N, k]\left(={ }_{N} C_{k}\right)$ are determined by the intrinsic $Z_{m}$ elements $\eta_{a \pm}^{(k)}$ and the representation matrices $U_{a}$ for the fundamental representation $\boldsymbol{N}=[N, 1]$. Here, $\Psi_{ \pm}$are six-dimensional Weyl fermions in $[N, k]$, and those boundary conditions are specified by representation matrices $T_{\Psi \pm}\left[U_{a}, \eta_{a \pm}^{(k)}\right]$, which

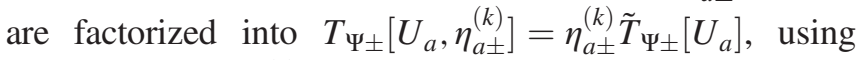
overall factors $\eta_{a \pm}^{(k)}$ intrinsic to fields and ${ }_{N} C_{k} \times{ }_{N} C_{k}$ matrices $\tilde{T}_{\Psi \pm}\left[U_{a}\right]$. Because $\mathcal{P}_{a \pm}^{(k)}$ are obtained as eigenval-

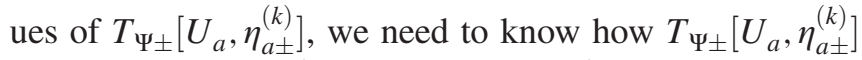
act as multiplets in $\left.{ }_{3} C_{l_{1}},{ }_{2} C_{l_{2}}, \ldots,{ }_{p_{n}} C_{l_{n}}\right)$. The components of $\Psi_{ \pm}$are written in the form of the antisymmetrization of the $k$-ple product of $N$, such as $[N, k]=(\boldsymbol{N} \times \ldots \times \boldsymbol{N})_{A}$, where a tiny subscript $A$ means the antisymmetrization, and the operation of $T_{\Psi_{ \pm}}\left[U_{a}, \eta_{a \pm}^{(k)}\right]$ on $[N, k]$ is given by $\eta_{a \pm}^{(k)}\left(\left(U_{a} \boldsymbol{N}\right) \times \ldots \times\left(U_{a} \boldsymbol{N}\right)\right)_{A}$. We consider a simple example of a $Z_{2}$ element with $U_{0}=\operatorname{diag}\left([+1]_{p_{1}},[-1]_{p_{2}}\right)$ where $[ \pm 1]_{p_{i}}$ represents \pm 1 for all $p_{i}$ elements. Then the $[N, k]$ of $S U(N)$ is decomposed into a sum of multiplets of $S U\left(p_{1}\right) \times S U\left(p_{2}\right)$ as $[N, k]=\sum_{l_{1}=0}^{k}\left({ }_{p_{1}} C_{l_{1}},{ }_{2} C_{l_{2}}\right)$, where $N=p_{1}+p_{2}$ and $k=l_{1}+l_{2}$. From the observation that $\left({ }_{p_{1}} C_{l_{1}},{ }_{2} C_{l_{2}}\right)$ is multiplied by $+1 l_{1}$ times and multiplied

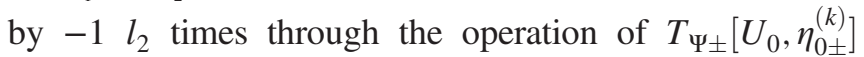
on $[N, k]$, we see the $Z_{2}$ element of $\left({ }_{p_{1}} C_{l_{1}}, p_{2} C_{l_{2}}\right)$ as $\mathcal{P}_{0 \pm}^{(k)}=\eta_{0 \pm}^{(k)}(+1)^{l_{1}}(-1)^{l_{2}}=(-1)^{l_{1}-k} \eta_{0 \pm}^{(k)}$, where we use $k=l_{1}+l_{2}$ and $(-1)^{n}=(-1)^{-n}$ ( $n$ is an integer). In this way, if $\eta_{a \pm}^{(k)}$ and $U_{a}$ are given, $\mathcal{P}_{a \pm}^{(k)}$ are determined for each multiplet, as will be done below.

We take the representation matrices for $T^{2} / Z_{2}$,

$$
\begin{aligned}
& U_{0}=\operatorname{diag}\left([+1]_{p_{1}},[+1]_{p_{2}},[+1]_{p_{3}},[+1]_{p_{4}},[-1]_{p_{5}},[-1]_{p_{6}},[-1]_{p_{7}},[-1]_{p_{8}}\right), \\
& U_{1}=\operatorname{diag}\left([+1]_{p_{1}},[+1]_{p_{2}},[-1]_{p_{3}},[-1]_{p_{4}},[+1]_{p_{5}},[+1]_{p_{6}},[-1]_{p_{7}},[-1]_{p_{8}}\right), \\
& U_{2}=\operatorname{diag}\left([+1]_{p_{1}},[-1]_{p_{2}},[+1]_{p_{3}},[-1]_{p_{4}},[+1]_{p_{5}},[-1]_{p_{6}},[+1]_{p_{7}},[-1]_{p_{8}}\right),
\end{aligned}
$$

where $[ \pm 1]_{p_{i}}$ represents \pm 1 for all $p_{i}$ elements. Then, the $Z_{2}$ elements $\mathcal{P}_{a \pm}^{(k)}$ of $\left({ }_{3} C_{l_{1}},{ }_{2} C_{l_{2}}, \ldots, p_{n} C_{l_{n}}\right)$ are determined as

$$
\begin{aligned}
& \mathcal{P}_{0 \pm}^{(k)}=(-1)^{l_{1}+l_{2}+l_{3}+l_{4}-k} \eta_{0 \pm}^{(k)}, \\
& \mathcal{P}_{1 \pm}^{(k)}=(-1)^{l_{1}+l_{2}+l_{5}+l_{6}-k} \eta_{1 \pm}^{(k)}, \\
& \mathcal{P}_{2 \pm}^{(k)}=(-1)^{l_{1}+l_{3}+l_{5}+l_{7}-k} \eta_{2 \pm}^{(k)} .
\end{aligned}
$$

In the same way, with the representation matrices for $T^{2} / Z_{3}$, 


$$
\begin{aligned}
& U_{0}=\operatorname{diag}\left([1]_{p_{1}},[1]_{p_{2}},[1]_{p_{3}},[\omega]_{p_{4}},[\omega]_{p_{5}},[\omega]_{p_{6}},[\bar{\omega}]_{p_{7}},[\bar{\omega}]_{p_{8}},[\bar{\omega}]_{p_{9}}\right), \\
& U_{1}=\operatorname{diag}\left([1]_{p_{1}},[\omega]_{p_{2}},[\bar{\omega}]_{p_{3}},[1]_{p_{4}},[\omega]_{p_{5}},[\bar{\omega}]_{p_{6}},[1]_{p_{7}},[\omega]_{p_{8}},[\bar{\omega}]_{p_{9}}\right),
\end{aligned}
$$

we obtain the relations

$$
\begin{aligned}
& \mathcal{P}_{0 \pm}^{(k)}=\omega^{l_{1}+l_{2}+l_{3}+2\left(l_{4}+l_{5}+l_{6}\right)-k} \eta_{0 \pm}^{(k)}, \\
& \mathcal{P}_{1 \pm}^{(k)}=\omega^{l_{1}+l_{4}+l_{7}+2\left(l_{2}+l_{5}+l_{8}\right)-k} \eta_{1 \pm}^{(k)} .
\end{aligned}
$$

With the representation matrices for $T^{2} / Z_{4}$,

$$
\begin{aligned}
& U_{0}=\operatorname{diag}\left([+1]_{p_{1}},[+1]_{p_{2}},[+i]_{p_{3}},[+i]_{p_{4}},[-1]_{p_{5}},[-1]_{p_{6}},[-i]_{p_{7}},[-i]_{p_{8}}\right), \\
& U_{1}=\operatorname{diag}\left([+1]_{p_{1}},[-1]_{p_{2}},[-i]_{p_{3}},[+i]_{p_{4}},[-1]_{p_{5}},[+1]_{p_{6}},[+i]_{p_{7}},[-i]_{p_{8}}\right),
\end{aligned}
$$

we obtain the relations

$$
\begin{aligned}
& \mathcal{P}_{0 \pm}^{(k)}=i^{l_{1}+l_{2}+2\left(l_{3}+l_{4}\right)+3\left(l_{5}+l_{6}\right)-k} \eta_{0 \pm}^{(k)}, \\
& \mathcal{P}_{1 \pm}^{(k)}=i^{l_{1}+l_{6}+2\left(l_{4}+l_{7}\right)+3\left(l_{2}+l_{5}\right)-k} \eta_{1 \pm}^{(k)} .
\end{aligned}
$$

With the representation matrix for $T^{2} / Z_{6}$,

$U_{0}=\operatorname{diag}\left([1]_{p_{1}},[\varphi]_{p_{2}},\left[\varphi^{2}\right]_{p_{3}},\left[\varphi^{3}\right]_{p_{4}},\left[\varphi^{4}\right]_{p_{5}},\left[\varphi^{5}\right]_{p_{6}}\right)$,

we obtain the relations

$$
\mathcal{P}_{0 \pm}^{(k)}=\varphi^{l_{1}+2 l_{2}+3 l_{3}+4 l_{4}+5 l_{5}-k} \eta_{0 \pm}^{(k)} .
$$

The subscripts $L$ and $R$ on the intrinsic $Z_{m}$ elements are omitted in (3.23), (3.25), (3.27), and (3.29). When we use ones with $L$ or $R, \eta_{a \pm R}^{(k)}$ are determined from $\eta_{a \pm L}^{(k)}$ as

$$
\eta_{a+R}^{(k)}=\rho \eta_{a+L}^{(k)}, \quad \eta_{a-R}^{(k)}=\bar{\rho} \eta_{a-L}^{(k)}
$$

as seen from (2.6). Intrinsic $Z_{m}$ elements satisfy the consistency conditions such as (A4), (A8), and the corresponding ones for $T^{2} / Z_{4}$ and $T^{2} / Z_{6}$. Hence the product of $\eta_{0 \pm}^{(k)}$ and $\eta_{1 \pm}^{(k)}$ should be 1 or -1 for $T^{2} / Z_{4}$.

In Appendix $\mathrm{C}$, we give formulas for flavor numbers from a fermion in $[N, k](=[N, N-k])$ and study the relationship between flavor numbers from a fermion in $\overline{[N, k]}$ and those from a fermion in $[N, k]$ from the viewpoint of charge conjugation.

\section{ORBIFOLD FAMILY UNIFICATION USING VECTORLIKE REPRESENTATION}

Now, we study whether or not three families of the SM matter multiplets are derived from a massless six-dimensional Dirac fermion (or a pair of six-dimensional Weyl fermions) in a vectorlike representation $[N, k]+[N, N-k]$ of $S U(N)$ $(N=8,9)$, through the orbifold breaking mechanism.

First, we explain that complete three SM families cannot be derived from a Dirac fermion in $[N, 1]+[N, N-1]$ or $[N, 2]+[N, N-2]$ of $S U(N)$ in our setup given in the previous section. After the breakdown of $S U(N), d_{R}$ and $\left(l_{L}\right)^{c}$ can appear from a Dirac fermion in $[N, 1]$ and $\left(d_{R}\right)^{c}$ and $l_{L}$ can appear from a Dirac fermion in $[N, N-1]$, but $q_{L}$, $\left(u_{R}\right)^{c}$, and $\left(e_{R}\right)^{c}$ cannot come from them. In the same way, after the breakdown of $S U(N)$, a Dirac fermion in $[N, 2]$ only generates one $q_{L}$, one $\left(u_{R}\right)^{c}$, and/or one $\left(e_{R}\right)^{c}$ at most, and that in $[N, N-2]$ only generates one $\left(q_{L}\right)^{c}$, one $u_{R}$, and/or one $e_{R}$ at most. Hence, a Dirac fermion in $[N, 3]+[N, N-3]$ has the smallest components among a possible candidate that produces three complete SM families.

Second, we present the total numbers of models with the three SM families, which originate from a Dirac fermion in $[N, 3]+[N, N-3]$ of $S U(8)$ and $S U(9)$. They are summarized in Table III. In Table III, the figures in parentheses represent numbers of models with three or more than three neutrino singlets. We list numbers $p_{i}(i=1, \ldots, 9)$ specifying representation matrices $U_{a}$ and the intrinsic $Z_{3}$ elements, to derive both the three families of the SM multiplets and the three neutrino singlets from a fermion in $[8,3]+[8,5]$ of $S U(8)$ on $M^{4} \times T^{2} / Z_{3}$, in Table IV. In Table IV, only the intrinsic $Z_{3}$ elements for the $\psi_{ \pm L}$ are written, and those for the $\psi_{ \pm R}$ can be seen from (3.30).

TABLE III. Total numbers of models with the three families of the SM multiplets.

\begin{tabular}{lccccc}
\hline \hline$S U(N)$ & Representations & $T^{2} / Z_{2}$ & $T^{2} / Z_{3}$ & $T^{2} / Z_{4}$ & $T^{2} / Z_{6}$ \\
\hline$S U(8)$ & {$[8,3]+[8,5]$} & $0(0)$ & $336(4)$ & $56(0)$ & $0(0)$ \\
$S U(9)$ & {$[9,3]+[9,6]$} & $1152(768)$ & $1188(600)$ & $512(416)$ & $0(0)$ \\
\hline \hline
\end{tabular}


TABLE IV. Models with the three families of the SM multiplets and three neutrino singlets from a fermion in $[8,3]+[8,5]$ on $M^{4} \times T^{2} / Z_{3}$.

\begin{tabular}{lcccc}
\hline \hline$\left(p_{1}, p_{2}, p_{3}, p_{4}, p_{5}, p_{6}, p_{7}, p_{8}, p_{9}\right)$ & $\left(\eta_{0+L}^{(3)}, \eta_{1+L}^{(3)}\right)$ & $\left(\eta_{0-L}^{(3)}, \eta_{1-L}^{(3)}\right)$ & $\left(\eta_{0+L}^{(5)}, \eta_{1+L}^{(5)}\right)$ & $\left(\eta_{0-L}^{(5)}, \eta_{1-L}^{(5)}\right)$ \\
\hline$(3,2,0,0,1,1,1,0,0)$ & $\left(\omega^{2}, 1\right)$ & $\left(\omega, \omega^{2}\right)$ & $\left(\omega^{2}, 1\right)$ & $\left(\omega, \omega^{2}\right)$ \\
$(3,2,0,0,1,1,1,0,0)$ & $\left(\omega^{2}, 1\right)$ & $\left(\omega^{2}, 1\right)$ & $\left(\omega^{2}, 1\right)$ & $\left(\omega^{2}, 1\right)$ \\
$(3,2,0,0,0,1,1,0,1)$ & $\left(\omega^{2}, 1\right)$ & $\left(\omega, \omega^{2}\right)$ & $\left(\omega^{2}, 1\right)$ & $\left(\omega, \omega^{2}\right)$ \\
$(3,2,0,0,0,1,1,0,1)$ & $\left(\omega, \omega^{2}\right)$ & $\left(\omega, \omega^{2}\right)$ & $\left(\omega, \omega^{2}\right)$ & $\left(\omega, \omega^{2}\right)$ \\
\hline \hline
\end{tabular}

Third, we give examples concerning the appearance of three SM families, using the first and second models in Table IV. By taking $\left(p_{1}, p_{2}, p_{3}, p_{4}, p_{5}, p_{6}, p_{7}, p_{8}, p_{9}\right)=$ $(3,2,0,0,1,1,1,0,0)$, the $S U(8)$ gauge symmetry is broken down as

$$
S U(8) \rightarrow S U(3)_{C} \times S U(2)_{L} \times U(1)^{4}
$$

Note that the residual gauge symmetry does not contain any non-Abelian continuous flavor symmetry. Then, $\mathbf{5 6}(=[8,3])$ and $\overline{\mathbf{5 6}}(=[8,5])$ are decomposed into particles with the SM gauge quantum numbers and its opposite ones, as shown in Tables V and VI, respectively. In the first and second columns, particles are denoted by using the symbols in the SM, and those with primes are regarded as mirror particles, which are particles with opposite quantum numbers under the SM gauge group. In the third column, any $l_{i}$ 's not on the list

TABLE V. Decomposition of $\mathbf{5 6}$ for $\left(p_{1}, p_{2}, p_{3}, p_{4}, p_{5}, p_{6}\right.$, $\left.p_{7}, p_{8}, p_{9}\right)=(3,2,0,0,1,1,1,0,0)$.

\begin{tabular}{|c|c|c|c|c|c|c|c|}
\hline & \\
\hline$\psi_{ \pm L}^{[8,3]}$ & $\psi_{ \pm R}^{[8,3]}$ & $\left(l_{1}, l_{2}, l_{5}, l_{6}, l_{7}\right)$ & $\left(\mathcal{P}_{0 \pm}^{(3)}, \mathcal{P}_{1 \pm}^{(3)}\right)$ & $\psi_{ \pm L}^{[8,5]}$ & $\psi_{ \pm R}^{[8,5]}$ & $\left(l_{1}, l_{2}, l_{5}, l_{6}, l_{7}\right)$ & $\left(\mathcal{P}_{0 \pm}^{(5)}, \mathcal{P}_{1 \pm}^{(5)}\right)$ \\
\hline$\left(e_{R}^{\prime}\right)^{c}$ & $e_{R}$ & $(3,0,0,0,0)$ & $\left(\eta_{0 \pm}^{(3)}, \eta_{1 \pm}^{(3)}\right)$ & $\left(e_{R}^{\prime}\right)^{c}$ & $e_{R}$ & $(3,0,1,1,0)$ & $\left(\omega^{2} \eta_{0 \pm}^{(5)}, \eta_{1 \pm}^{(5)}\right)$ \\
\hline$q_{L}^{\prime}$ & $\left(q_{L}\right)^{c}$ & $(2,1,0,0,0)$ & $\left(\eta_{0 \pm}^{(3)}, \omega \eta_{1 \pm}^{(3)}\right)$ & & & $(3,0,1,0,1)$ & $\left(\eta_{0 \pm}^{(5)}, \omega \eta_{1 \pm}^{(5)}\right)$ \\
\hline$\left(u_{R}^{\prime}\right)^{c}$ & $u_{R}$ & $(1,2,0,0,0)$ & $\left(\eta_{0 \pm}^{(3)}, \omega^{2} \eta_{1 \pm}^{(3)}\right)$ & & & $(3,0,0,1,1)$ & $\left(\eta_{0 \pm}^{(5)}, \omega^{2} \eta_{1 \pm}^{(5)}\right)$ \\
\hline$\left(u_{R}\right)^{c}$ & $u_{R}^{\prime}$ & $(2,0,1,0,0)$ & $\left(\omega \eta_{0+}^{(3)}, \omega \eta_{1+}^{(3)}\right)$ & $q_{L}^{\prime}$ & $\left(q_{L}\right)^{c}$ & $(2,1,1,1,0)$ & $\left(\omega^{2} \eta_{0 \pm}^{(5)}, \omega \eta_{1 \pm}^{(5)}\right)$ \\
\hline & & $(2,0,0,1,0)$ & $\left(\omega \eta_{0 \pm}^{(3)}, \omega^{2} \eta_{1 \pm}^{(3)}\right)$ & & & $(2,1,1,0,1)$ & $\left(\eta_{0 \pm}^{(5)}, \omega^{2} \eta_{1 \pm}^{(5)}\right)$ \\
\hline & & $(2,0,0,0,1)$ & $\left(\omega^{2} \eta_{0 \pm}^{(3)}, \eta_{1 \pm}^{(3)}\right)$ & & & $(2,1,0,1,1)$ & $\left(\eta_{0 \pm}^{(5)}, \eta_{1 \pm}^{(5)}\right)$ \\
\hline$q_{L}$ & $\left(q_{L}^{\prime}\right)^{c}$ & $(1,1,1,0,0)$ & $\left(\omega \eta_{0+}^{(3)}, \omega^{2} \eta_{1+}^{(3)}\right)$ & $\left(u_{R}^{\prime}\right)^{c}$ & $u_{R}$ & $(1,2,1,1,0)$ & $\left(\omega^{2} \eta_{0+}^{(5)}, \omega^{2} \eta_{1+}^{(5)}\right)$ \\
\hline & & $(1,1,0,1,0)$ & $\left(\omega \eta_{0 \pm}^{(3)}, \eta_{1 \pm}^{(3)}\right)$ & & & $(1,2,1,0,1)$ & $\left(\eta_{0 \pm}^{(5)}, \eta_{1 \pm}^{(5)}\right)$ \\
\hline & & $(1,1,0,0,1)$ & $\left(\omega^{2} \eta_{0 \pm}^{(3)}, \omega \eta_{1 \pm}^{(3)}\right)$ & & & $(1,2,0,1,1)$ & $\left(\eta_{0 \pm}^{(5)}, \omega \eta_{1 \pm}^{(5)}\right)$ \\
\hline$\left(e_{R}\right)^{c}$ & $e_{R}^{\prime}$ & $(0,2,1,0,0)$ & $\left(\omega \eta_{0 \pm}^{(3)}, \eta_{1 \pm}^{(3)}\right)$ & $\left(u_{R}\right)^{c}$ & $u_{R}^{\prime}$ & $(2,0,1,1,1)$ & $\left(\omega \eta_{0 \pm}^{(5)}, \eta_{1 \pm}^{(5)}\right)$ \\
\hline & & $(0,2,0,1,0)$ & $\left(\omega \eta_{0 \pm}^{(3)}, \omega \eta_{1 \pm}^{(3)}\right)$ & $q_{L}$ & $\left(q_{L}^{\prime}\right)^{c}$ & $(1,1,1,1,1)$ & $\left(\omega \eta_{0 \pm}^{(5)}, \omega \eta_{1 \pm}^{(5)}\right)$ \\
\hline & & $(0,2,0,0,1)$ & $\left(\omega^{2} \eta_{0 \pm}^{(3)}, \omega^{2} \eta_{1 \pm}^{(3)}\right)$ & $\left(e_{R}\right)^{c}$ & $e_{R}^{\prime}$ & $(0,2,1,1,1)$ & $\left(\omega \eta_{0 \pm}^{(5)}, \omega^{2} \eta_{1 \pm}^{(5)}\right)$ \\
\hline$\left(d_{R}^{\prime}\right)^{c}$ & $d_{R}$ & $(1,0,1,1,0)$ & $\left(\omega^{2} \eta_{0 \pm}^{(3)}, \eta_{1 \pm}^{(3)}\right)$ & $\left(d_{R}\right)^{c}$ & $d_{R}^{\prime}$ & $(2,2,1,0,0)$ & $\left(\omega \eta_{0 \pm}^{(5)}, \eta_{1 \pm}^{(5)}\right)$ \\
\hline & & $(1,0,1,0,1)$ & $\left(\eta_{0 \pm}^{(3)}, \omega \eta_{1 \pm}^{(3)}\right)$ & & & $(2,2,0,1,0)$ & $\left(\omega \eta_{0 \pm}^{(5)}, \omega \eta_{1 \pm}^{(5)}\right)$ \\
\hline & & $(1,0,0,1,1)$ & $\left(\eta_{0 \pm}^{(3)}, \omega^{2} \eta_{1 \pm}^{(3)}\right)$ & & & $(2,2,0,0,1)$ & $\left(\omega^{2} \eta_{0 \pm}^{(5)}, \omega^{2} \eta_{1 \pm}^{(5)}\right)$ \\
\hline$l_{L}^{\prime}$ & $\left(l_{L}\right)^{c}$ & $(0,1,1,1,0)$ & $\left(\omega^{2} \eta_{0 \pm}^{(3)}, \omega \eta_{1 \pm}^{(3)}\right)$ & $l_{L}$ & $\left(l_{L}^{\prime}\right)^{c}$ & $(3,1,1,0,0)$ & $\left(\omega \eta_{0 \pm}^{(5)}, \omega^{2} \eta_{1 \pm}^{(5)}\right)$ \\
\hline & & $(0,1,1,0,1)$ & $\left(\eta_{0 \pm}^{(3)}, \omega^{2} \eta_{1 \pm}^{(3)}\right)$ & & & $(3,1,0,1,0)$ & $\left(\omega \eta_{0 \pm}^{(5)}, \eta_{1 \pm}^{(5)}\right)$ \\
\hline & & $(0,1,0,1,1)$ & $\left(\eta_{0+}^{(3)}, \eta_{1+}^{(3)}\right)$ & & & $(3,1,0,0,1)$ & $\left(\omega^{2} \eta_{0 \pm}^{(5)}, \omega \eta_{1 \pm}^{(5)}\right)$ \\
\hline$\left(\nu_{R}\right)^{c}$ & $\nu_{R}$ & $(0,0,1,1,1)$ & $\left(\omega \eta_{0 \pm}^{(3)}, \eta_{1 \pm}^{(3)}\right)$ & $\left(\nu_{R}\right)^{c}$ & $\nu_{R}$ & $(3,2,0,0,0)$ & $\left(\eta_{0 \pm}^{(5)}, \omega^{2} \eta_{1 \pm}^{(5)}\right)$ \\
\hline
\end{tabular}

are zero. In the fourth column, the subscripts $L$ and $R$ are omitted on the intrinsic $Z_{3}$ elements.

We give an assignment of intrinsic $Z_{3}$ elements and particle contents to derive three SM families and three neutrino singlets as zero modes in Table VII. As seen from Table VII, just three sets of SM fermions $\left[q_{L}^{i},\left(u_{R}^{i}\right)^{c},\left(d_{R}^{i}\right)^{c}, l_{L}^{i},\left(e_{R}^{i}\right)^{c}\right]$ and three kinds of neutrino singlets $\left[\left(\nu_{R}\right)^{c}\right.$ and $\left.\nu^{R}\right]$ are originated as zero modes from $\psi_{ \pm L}^{[8,3]}+\psi_{ \pm R}^{[8,3]}+\psi_{ \pm L}^{[8,5]}+\psi_{ \pm R}^{[8,5]}$ with suitable intrinsic $Z_{3}$ elements, using the survival hypothesis. Mirror particles can disappear by acquiring heavy masses, that is, the $l_{L}^{\prime}$ in $\psi_{-L}^{[8,3]}$ can be massive with one of $l_{L},\left(l_{L}\right)^{c}$ or a mixture of them and the $q_{L}^{\prime}$ in $\psi_{-L}^{[8,5]}$ can be massive with one of $q_{L}$, $\left(q_{L}\right)^{c}$ or a mixture of them.

In the same way, we can obtain particle contents with just three SM families and three neutrino singlets as zero modes

TABLE VI. Decomposition of $\overline{\mathbf{5 6}}$ for $\left(p_{1}, p_{2}, p_{3}, p_{4}, p_{5}\right.$, $\left.p_{6}, p_{7}, p_{8}, p_{9}\right)=(3,2,0,0,1,1,1,0,0)$. 
TABLE VII. The particle contents as zero modes obtained from 56 and $\overline{\mathbf{5 6}}$.

\begin{tabular}{lccccccc}
\hline Multiplets & $\left(\eta_{0 \pm}^{(k)}, \eta_{1 \pm}^{(k)}\right)$ & $\left(d_{R}\right)^{c}$ & $l_{L}$ & $\left(u_{R}\right)^{c}$ & $\left(e_{R}\right)^{c}$ & $q_{L}$ & $\left(\nu_{R}\right)^{c}$ \\
\hline$\psi_{+L}^{[8,3]}$ & $\left(\omega^{2}, 1\right)$ & & & & $\left(e_{R}\right)^{c}$ & $q_{L}$ & $\left(\nu_{R}\right)^{c}$ \\
$\psi_{+R}^{[8,3]}$ & $(1, \omega)$ & $d_{R}$ & $\left(l_{L}\right)^{c}$ & $u_{R}$ & & & \\
$\psi_{-L}^{[8,3]}$ & $\left(\omega, \omega^{2}\right)$ & & $l_{L}^{\prime}$ & & & $q_{L}$ & \\
$\psi_{-R}^{[8,3]}$ & $(1, \omega)$ & $d_{R}$ & $\left(l_{L}\right)^{c}$ & $u_{R}$ & & & \\
$\psi_{+L}^{[8,5]}$ & $\left(\omega^{2}, 1\right)$ & $\left(d_{R}\right)^{c}$ & $l_{L}$ & $\left(u_{R}\right)^{c}$ & & & \\
$\psi_{+R}^{[8,5]}$ & $(1, \omega)$ & & & & $e_{R}$ & $\left(q_{L}\right)^{c}$ & $\nu_{R}$ \\
$\psi_{-L}^{[8,5]}$ & $\left(\omega, \omega^{2}\right)$ & & $l_{L}$ & & & $q_{L}^{\prime}$ & \\
$\psi_{-R}^{[8,5]}$ & $(1, \omega)$ & & & & $e_{R}$ & $\left(q_{L}\right)^{c}$ & $\nu_{R}$ \\
\hline \hline
\end{tabular}

TABLE VIII. Another assignment of intrinsic $Z_{3}$ elements and the particle contents as zero modes obtained from 56 and $\overline{\mathbf{5 6}}$.

\begin{tabular}{lccccccc}
\hline \hline Multiplets & $\left(\eta_{0 \pm}^{(k)}, \eta_{1 \pm}^{(k)}\right)$ & $\left(d_{R}\right)^{c}$ & $l_{L}$ & $\left(u_{R}\right)^{c}$ & $\left(e_{R}\right)^{c}$ & $q_{L}$ & $\left(\nu_{R}\right)^{c}$ \\
\hline$\psi_{+L}^{[8,3]}$ & $\left(\omega^{2}, 1\right)$ & & & & $\left(e_{R}\right)^{c}$ & $q_{L}$ & $\left(\nu_{R}\right)^{c}$ \\
$\psi_{+R}^{[8,3]}$ & $(1, \omega)$ & $d_{R}$ & $\left(l_{L}\right)^{c}$ & $u_{R}$ & & & \\
$\psi_{-L}^{[8,3]}$ & $\left(\omega^{2}, 1\right)$ & & & & $\left(e_{R}\right)^{c}$ & $q_{L}$ & $\left(\nu_{R}\right)^{c}$ \\
$\psi_{-R}^{[8,3]}$ & $\left(\omega, \omega^{2}\right)$ & & $\left(l_{L}\right)^{c}$ & & & $\left(q_{L}^{\prime}\right)^{c}$ & \\
$\psi_{+L}^{[8,5]}$ & $\left(\omega^{2}, 1\right)$ & $\left(d_{R}\right)^{c}$ & $l_{L}$ & $\left(u_{R}\right)^{c}$ & & & \\
$\psi_{+R}^{[8,5]}$ & $(1, \omega)$ & & & & $e_{R}$ & $\left(q_{L}\right)^{c}$ & $\nu_{R}$ \\
$\psi_{-L}^{[8,5]}$ & $\left(\omega^{2}, 1\right)$ & $\left(d_{R}\right)^{c}$ & $l_{L}$ & $\left(u_{R}\right)^{c}$ & & & \\
$\psi_{-R}^{[8,5]}$ & $\left(\omega, \omega^{2}\right)$ & & $\left(l_{L}^{\prime}\right)^{c}$ & & & $\left(q_{L}\right)^{c}$ & \\
\hline \hline
\end{tabular}

from $\psi_{ \pm L}^{[8,3]}+\psi_{ \pm R}^{[8,3]}+\psi_{ \pm L}^{[8,5]}+\psi_{ \pm R}^{[8,5]}, \quad$ with intrinsic $Z_{3}$ elements assigned in Table VIII, after the survival hypothesis works.

Finally, we point out that the classification of our models has not yet been completed in our setup. Concretely, we consider the breaking pattern (2.9), with the identification of $S U\left(p_{1}\right)=S U(3)_{C}$ and $S U\left(p_{2}\right)=S U(2)_{L}$, and take the diagonal representation matrices (3.22), (3.24), (3.26), and (3.28). Based on the representation matrices given above, there is a choice to take $p_{i}=3$ and $p_{j}=2$ with $(i, j) \neq(1,2)$ as $S U(3)_{C} \times S U(2)_{L}$. Or provided that $p_{1}=3$ and $p_{2}=2$, we can choose different diagonal representation matrices, that are obtained by the exchange of components in the above ones. The same results are obtained from most of them, but there are independent choices to generate models different from those mentioned in this section. Complete analysis and classification will be reported, including results from a fermion in $[N, k]+$ $[N, N-k](k \geq 4)$, in a forthcoming paper [47].

\section{CONCLUSIONS}

We have studied the possibility of family unification on the basis of $S U(N)$ gauge theory in the six-dimensional space-time $M^{4} \times T^{2} / Z_{m}(m=2,3,4,6)$. We have obtained enormous numbers of models with three families of the SM matter multiplets derived from a massless six-dimensional Dirac fermion in a vectorlike representation $[N, 3]+$ $[N, N-3]$ of $S U(N)(N=8,9)$, through the orbifold breaking mechanism, and found models with three or more than three neutrino singlets and without any non-Abelian continuous flavor gauge symmetries. We have shown a feature that each flavor number from a fermion in $[N, k]$ with intrinsic $Z_{m}$ elements $\eta_{a \pm}^{(k)}$ is equal to that from a fermion in $\overline{[N, k]}(=[N, N-k])$ with appropriate $\eta_{a \pm}^{(N-k)}$, because there is a one-to-one correspondence between zero modes from a Weyl fermion in $[N, k]$ with $\eta_{a \pm}^{(k)}$ and those from a Weyl fermion in $[N, N-k]$ with appropriate $\eta_{a \pm}^{(N-k)}$, using the equivalence under the charge conjugation.

Now, we have several problems as a future work.

It is meaningful to study phenomenological implications relating to the breakdown of extra $U(1)$ gauge symmetries; $D$-term contributions to scalar (squark, slepton, and Higgs) masses; and the generation of realistic fermion masses and family mixing, based on $S U(8)$ models illustrated in Sec. IV. The $S U(8)$ models are attractive, because there is no non-Abelian continuous gauge group, and extra $U(1)$ gauge bosons can be massive by the vacuum expectation values of the SM singlets scalar fields. Moreover, superpartners of neutrino singlets can be candidates of such scalar fields. In SUSY models, there appear $D$-term contributions to scalar masses after the breakdown of extra gauge symmetries, if soft SUSY breaking terms have a nonuniversal structure, and its contributions lift the mass degeneracy [48-52]. Under assumptions that SUSY is broken down by the dynamics on a brane and nonuniversal soft SUSY breaking terms are induced, the $D$-term contributions have been studied in the framework of $S U(N)$ orbifold GUTs [53-55], and they can become useful probes to specify a realistic model in GUTs. Then we need to reconsider the anomaly cancellations on a construction of SUSY models, because various fermions exist there. Fermion mass hierarchy and family mixing can occur through the Froggatt-Nielsen mechanism [56] on the breakdown of extra $U(1)$ gauge symmetries and/or the suppression of brane-localized Yukawa coupling constants among brane weak Higgs doublets and bulk fermions with the volume suppression factor [57].

It would be interesting to reconstruct our models in the framework of $E_{8}$ gauge theory or superstring theory. Various four-dimensional string models including three families have been constructed from several methods; see, e.g., [58] and references therein for useful articles. ${ }^{6}$ It has been pointed out that $S O(1, D-1)$ space-time symmetry can lead to family structure $[61,62]$, and hence it would offer a hint to explore the family structure in our models.

\footnotetext{
${ }^{6}$ See also Refs. $[59,60]$ and references therein for recent works.
} 
Furthermore, it would be intriguing to study cosmological implications of the class of models presented in this paper; see, e.g., [63] and references therein for useful articles toward this direction.

\section{ACKNOWLEDGMENTS}

This work was supported in part by scientific grants from Iwanami Fu-Jukai and the MEXT-Supported Program for the Strategic Research Foundation at Private Universities Topological Science under Grant No. S1511006 (Y. G.) and from the Ministry of Education, Culture, Sports, Science and Technology under Grant No. 17K05413 (Y. K.).

\section{APPENDIX A: $T^{2} / Z_{m}$ ORBIFOLD}

\section{1. $T^{2} / Z_{2}$}

The orbifold $T^{2} / Z_{2}$ is obtained by identifying $z+e_{1}$, $z+e_{2}$, and $-z$ with $z$. Here $e_{1}=1$ and $e_{2}=i$. The resultant space is depicted in Fig. 1. Fixed points $z_{\mathrm{fp}}$ satisfy $z_{\mathrm{fp}}=-z_{\mathrm{fp}}+a e_{1}+b e_{2}$, where $a$ and $b$ are integers. There are four kinds of fixed points: $0, e_{1} / 2, e_{2} / 2$, and $\left(e_{1}+e_{2}\right) / 2$. Around these points, we define six kinds of transformations:

$s_{0}: z \rightarrow-z, \quad s_{1}: z \rightarrow-z+e_{1}$,

$s_{2}: z \rightarrow-z+e_{2}, \quad s_{3}: z \rightarrow-z+e_{1}+e_{2}$,

$t_{1}: z \rightarrow z+e_{1}, \quad t_{2}: z \rightarrow z+e_{2}$,

and they satisfy the relations

$s_{0}^{2}=s_{1}^{2}=s_{2}^{2}=s_{3}^{2}=I, \quad s_{1}=t_{1} s_{0}, \quad s_{2}=t_{2} s_{0}$,

$s_{3}=t_{1} t_{2} s_{0}=s_{1} s_{0} s_{2}=s_{2} s_{0} s_{1}, \quad t_{1} t_{2}=t_{2} t_{1}$,

where $I$ is the identity operation.

The boundary conditions of six-dimensional bulk fields are specified by representation matrices $\left(U_{0}, U_{1}, U_{2}, U_{3}\right.$, $\left.V_{1}, V_{2}\right)$ and intrinsic $Z_{2}$ elements $\left(\eta_{0}, \eta_{1}, \eta_{2}, \eta_{3}, \xi_{1}, \xi_{2}\right)$

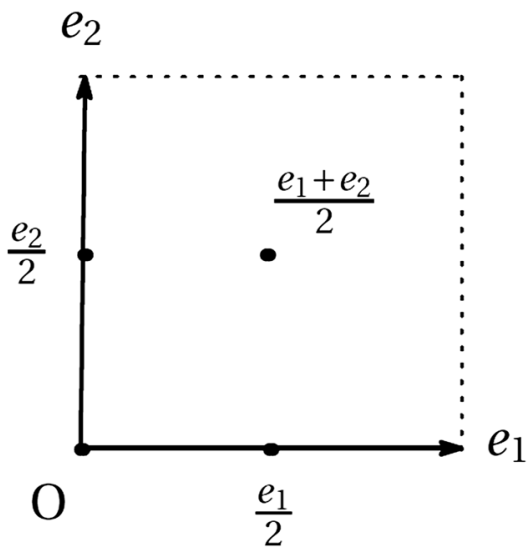

FIG. 1. Orbifold $T^{2} / Z_{2}$. corresponding to the above transformations. These matrices and $Z_{2}$ elements satisfy the relations

$U_{0}^{2}=U_{1}^{2}=U_{2}^{2}=U_{3}^{2}=I, \quad U_{1}=V_{1} U_{0}, \quad U_{2}=V_{2} U_{0}$,
$U_{3}=V_{1} V_{2} U_{0}=U_{1} U_{0} U_{2}=U_{2} U_{0} U_{1}, \quad V_{1} V_{2}=V_{2} V_{1}$,

$$
\begin{aligned}
& \eta_{0}^{2}=\eta_{1}^{2}=\eta_{2}^{2}=\eta_{3}^{2}=1, \quad \eta_{1}=\xi_{1} \eta_{0} \\
& \eta_{2}=\xi_{2} \eta_{0}, \quad \eta_{3}=\xi_{1} \xi_{2} \eta_{0}=\eta_{1} \eta_{0} \eta_{2},
\end{aligned}
$$

as the consistency conditions. Here, we omit the subscripts specifying fields and/or chiralities such as $\Phi, \pm, L$, and/or $R$. Note that $\eta_{1} \eta_{0} \eta_{2}=\eta_{2} \eta_{0} \eta_{1}$ and $\xi_{1} \xi_{2}=\xi_{2} \xi_{1}$ hold automatically because intrinsic $Z_{m}$ elements are numbers. From (A2) and (A3), we find that any three transformations are independent and others are constructed as combinations of them. We choose the transformations $s_{0}: z \rightarrow-z$, $s_{1}: z \rightarrow 1-z$, and $s_{2}: z \rightarrow i-z$ and the corresponding matrices $U_{0}, U_{1}$, and $U_{2}$.

\section{2. $T^{2} / Z_{3}$}

The orbifold $T^{2} / Z_{3}$ is obtained by identifying $z+e_{1}$, $z+e_{2}$, and $\omega z$ with $z$. Here $e_{1}=1$ and $e_{2}=\omega=e^{2 \pi i / 3}$. The resultant space is depicted in Fig. 2. Fixed points satisfying $z_{\mathrm{fp}}=\omega z_{\mathrm{fp}}+a e_{1}+b e_{2}$ (where $a, b$ are integers) are $z=0,\left(2 e_{1}+e_{2}\right) / 3$, and $\left(e_{1}+2 e_{2}\right) / 3$. Around these points, we define five kinds of transformations:

$$
\begin{aligned}
& s_{0}: z \rightarrow \omega z, \quad s_{1}: z \rightarrow \omega z+e_{1}, \\
& s_{2}: z \rightarrow \omega z+e_{1}+e_{2}, \\
& t_{1}: z \rightarrow z+e_{1}, \quad t_{2}: z \rightarrow z+e_{2},
\end{aligned}
$$

and they satisfy the relations

$$
\begin{aligned}
& s_{0}^{3}=s_{1}^{3}=s_{2}^{3}=s_{0} s_{1} s_{2}=s_{1} s_{2} s_{0}=s_{2} s_{0} s_{1}=I, \\
& s_{1}=t_{1} s_{0}, \quad s_{2}=t_{2} t_{1} s_{0}, \quad t_{1} t_{2}=t_{2} t_{1} .
\end{aligned}
$$

The boundary conditions of bulk fields are specified by matrices $\left(U_{0}, U_{1}, U_{2}, V_{1}, V_{2}\right)$ and intrinsic $Z_{3}$ elements $\left(\eta_{0}, \eta_{1}, \eta_{2}, \xi_{1}, \xi_{2}\right)$ satisfying the relations

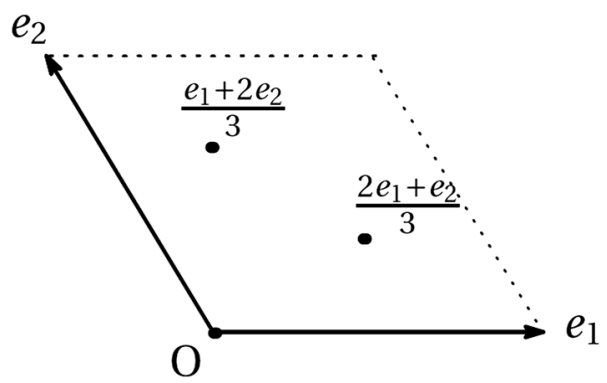

FIG. 2. Orbifold $T^{2} / Z_{3}$. 


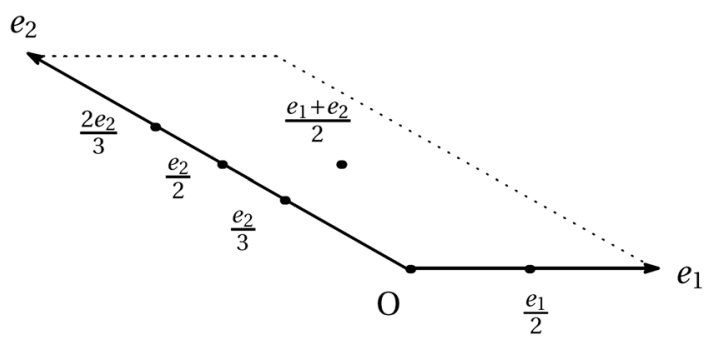

FIG. 3. Orbifold $T^{2} / Z_{6}$.

$U_{0}^{3}=U_{1}^{3}=U_{2}^{3}=U_{0} U_{1} U_{2}=U_{1} U_{2} U_{0}=U_{2} U_{0} U_{1}=I$,

$U_{1}=V_{1} U_{0}, \quad U_{2}=V_{2} V_{1} U_{0}, \quad V_{1} V_{2}=V_{2} V_{1}$,

$\eta_{0}^{3}=\eta_{1}^{3}=\eta_{2}^{3}=\eta_{0} \eta_{1} \eta_{2}=1, \quad \eta_{1}=\xi_{1} \eta_{0}, \quad \eta_{2}=\xi_{2} \xi_{1} \eta_{0}$,

where we omit the subscripts specifying fields and/or chiralities such as $\Phi, \pm, L$, and/or $R$. Because two of these matrices are independent, we choose representation matrices $U_{0}$ and $U_{1}$ corresponding to the transformations $s_{0}: z \rightarrow e^{2 \pi i / 3} z$ and $s_{1}: z \rightarrow e^{2 \pi i / 3} z+1$.

\section{3. $T^{2} / Z_{4}$}

The orbifold $T^{2} / Z_{4}$ is obtained by identifying $z+e_{1}$, $z+e_{2}, i z$, and $-z$ with $z$. Here $e_{1}=1$ and $e_{2}=i$. The resultant space is depicted as the same figure as $T^{2} / Z_{2}$. Fixed points are $z_{\mathrm{fp}}=0$ and $\left(e_{1}+e_{2}\right) / 2$ for the $Z_{4}$ transformation $z \rightarrow i z$ and $z_{\mathrm{fp}}=0, e_{1} / 2, e_{2} / 2$, and $\left(e_{1}+e_{2}\right) / 2$ for the $Z_{2}$ transformation $z \rightarrow-z$. Around these points, we define eight kinds of transformations:

$$
\begin{aligned}
s_{0}: z & \rightarrow i z, \quad s_{1}: z \rightarrow i z+e_{1}, \quad s_{20}: z \rightarrow-z, \\
s_{21}: z & \rightarrow-z+e_{1}, \quad s_{22}: z \rightarrow-z+e_{2}, \\
s_{23}: z & \rightarrow-z+e_{1}+e_{2}, \\
t_{1}: z & \rightarrow z+e_{1}, \quad t_{2}: z \rightarrow z+e_{2}
\end{aligned}
$$

and they satisfy the relations

$$
\begin{aligned}
s_{0}^{4} & =s_{1}^{4}=s_{20}^{2}=s_{21}^{2}=s_{22}^{2}=s_{23}^{2}=I, \\
s_{1} & =t_{1} s_{0}, \quad s_{21}=t_{1} s_{20}, \\
s_{22} & =t_{2} s_{20}, \quad s_{20}=s_{0}^{2}, \quad s_{21}=s_{1} s_{0}, \quad s_{22}=s_{0} s_{1}, \\
s_{23} & =t_{1} t_{2} s_{20}=s_{21} s_{20} s_{22}=s_{22} s_{20} s_{21}, \quad t_{1} t_{2}=t_{2} t_{1} .
\end{aligned}
$$

The $Z_{4}$ transformations $s_{0}$ and $s_{1}$ are independent of each other and those representation matrices are denoted as $U_{0}$ and $U_{1}$, respectively. Other representation matrices are determined uniquely, if $U_{0}$ and $U_{1}$ are given.

\section{4. $T^{2} / Z_{6}$}

$T^{2}$ is constructed by the $G_{2}$ lattice whose basis vectors are $e_{1}=1$ and $e_{2}=(-3+i \sqrt{3}) / 2$. The orbifold $T^{2} / Z_{6}$ is obtained by further identifying $\varphi z$ with $z$ where $\varphi=e^{\pi i / 3}$. The resultant space is depicted in Fig. 3. Basis vectors are transformed as $\varphi e_{1}=2 e_{1}+e_{2}, \varphi e_{2}=-3 e_{1}-e_{2}$ under the $Z_{6}$ transformation $z \rightarrow \varphi z$. Fixed points are $z_{\mathrm{fp}}=0$ for the $Z_{6}$ transformation $z \rightarrow \varphi z ; z_{\mathrm{fp}}=0, e_{2} / 3$, and $2 e_{2} / 3$ for the $Z_{3}$ transformation $z \rightarrow \varphi^{2} z$; and $z_{\mathrm{fp}}=0, e_{1} / 2, e_{2} / 2$, and $\left(e_{1}+e_{2}\right) / 2$ for the $Z_{2}$ transformation $z \rightarrow \varphi^{3} z$. Around these points we define ten kinds of transformations:

$$
\begin{aligned}
s_{0}: z & \rightarrow \varphi z, \quad s_{10}: z \rightarrow \varphi^{2} z, \quad s_{11}: z \rightarrow \varphi^{2} z+e_{1}+e_{2}, \quad s_{12}: z \rightarrow \varphi^{2} z+2 e_{1}+2 e_{2}, \\
s_{20}: z & \rightarrow \varphi^{3} z, \quad s_{21}: z \rightarrow \varphi^{3} z+e_{1}, \quad s_{22}: z \rightarrow \varphi^{3} z+e_{2}, \quad s_{23}: z \rightarrow \varphi^{3} z+e_{1}+e_{2}, \\
t_{1}: z & \rightarrow z+e_{1}, \quad t_{2}: z \rightarrow z+e_{2},
\end{aligned}
$$

and they satisfy the relations

$$
\begin{aligned}
s_{0}^{6} & =s_{10}^{3}=s_{11}^{3}=s_{12}^{3}=s_{20}^{2}=s_{21}^{2}=s_{22}^{2}=s_{23}^{2}=I, \quad s_{11}=t_{1} t_{2} s_{10}, \quad s_{12}=t_{1}^{2} t_{2}^{2} s_{10}, \\
s_{21} & =t_{1} s_{20}, \quad s_{22}=t_{2} s_{20}, \quad s_{23}=t_{1} t_{2} s_{20}=s_{21} s_{20} s_{22}=s_{22} s_{20} s_{21}=s_{11} s_{0}, \\
s_{10} & =s_{0}^{2}, \quad s_{20}=s_{0}^{3}, \quad t_{1} t_{2}=t_{2} t_{1}, \quad t_{2}=s_{0}^{2} t_{1} s_{0} t_{1} s_{0}^{3}, \\
\left(s_{0} s_{10}\right)^{4} & =\left(s_{0} s_{11}\right)^{4}=\left(s_{0} s_{12}\right)^{4}=I, \quad\left(s_{0} s_{20}\right)^{3}=\left(s_{0} s_{21}\right)^{3}=\left(s_{0} s_{22}\right)^{3}=\left(s_{0} s_{23}\right)^{3}=I .
\end{aligned}
$$

We denote the representation matrix for the $Z_{6}$ transformation $s_{0}: z \rightarrow e^{\pi i / 3} z$ as $U_{0}$ and other representation matrices are determined uniquely, if $U_{0}$ is given.

\section{APPENDIX B: FERMIONS ON SIX DIMENSIONS}

We explain gamma matrices, charge conjugation of fermions, and $Z_{m}$ transformation properties in six 
dimensions [64]. We use the metric $\eta_{M N}=\operatorname{diag}(1,-1,-1$, $-1,-1,-1)(M, N=0,1,2,3,5,6)$, and the following representation for six-dimensional gamma matrices:

$$
\begin{aligned}
& \Gamma^{\mu}=\gamma^{\mu} \otimes \sigma^{3}=\left(\begin{array}{cc}
\gamma^{\mu} & 0 \\
0 & -\gamma^{\mu}
\end{array}\right), \\
& \Gamma^{5}=I_{4 \times 4} \otimes i \sigma^{1}=\left(\begin{array}{cc}
0 & i I_{4 \times 4} \\
i I_{4 \times 4} & 0
\end{array}\right), \\
& \Gamma^{6}=I_{4 \times 4} \otimes i \sigma^{2}=\left(\begin{array}{cc}
0 & I_{4 \times 4} \\
-I_{4 \times 4} & 0
\end{array}\right),
\end{aligned}
$$

where $\mu=0,1,2,3, \sigma^{i}(i=1,2,3)$ are Pauli matrices, and $I_{4 \times 4}$ is the $4 \times 4$ unit matrix. We take the chiral representation on four-dimensional space-time for $\gamma^{\mu}$ such that

$\gamma^{\mu} \equiv\left(\begin{array}{cc}0 & \sigma^{\mu} \\ \bar{\sigma}^{\mu} & 0\end{array}\right), \quad \sigma^{\mu}=\left(I_{2 \times 2}, \sigma^{i}\right), \quad \bar{\sigma}^{\mu}=\left(I_{2 \times 2},-\sigma^{i}\right)$,

where $I_{2 \times 2}$ is the $2 \times 2$ unit matrix. The $\Gamma^{M}$ satisfy the anticommutation relations of the Clifford algebra such that $\left\{\Gamma^{M}, \Gamma^{N}\right\}=2 \eta^{M N}$, where $\eta^{M N}$ is the inverse of $\eta_{M N}$. The chirality operator $\Gamma_{7}$ for the six-dimensional fermion $\Psi$ is defined by

$\Gamma_{7} \equiv \Gamma^{0} \Gamma^{1} \Gamma^{2} \Gamma^{3} \Gamma^{5} \Gamma^{6}=-\gamma_{5} \otimes \sigma^{3}=\left(\begin{array}{cc}-\gamma_{5} & 0 \\ 0 & \gamma_{5}\end{array}\right)$,

where $\gamma_{5}$ is the chirality operator in four dimensions defined by

$$
\gamma_{5} \equiv i \gamma^{0} \gamma^{1} \gamma^{2} \gamma^{3} \equiv\left(\begin{array}{cc}
-I_{2 \times 2} & 0 \\
0 & I_{2 \times 2}
\end{array}\right)
$$

Six-dimensional fermions with a definite chirality are called Weyl fermions in six dimensions. The Weyl fermion $\left(\Psi_{+}\right)$with positive chirality and that $\left(\Psi_{-}\right)$with negative chirality are given by

$$
\begin{aligned}
& \Psi_{+}=\frac{1+\Gamma_{7}}{2} \Psi=\left(\begin{array}{cc}
\frac{1-\gamma_{5}}{2} & 0 \\
0 & \frac{1+\gamma_{5}}{2}
\end{array}\right) \Psi=\left(\begin{array}{l}
\psi_{+L} \\
\psi_{+R}
\end{array}\right), \\
& \Psi_{-}=\frac{1-\Gamma_{7}}{2} \Psi=\left(\begin{array}{cc}
\frac{1+\gamma_{5}}{2} & 0 \\
0 & \frac{1-\gamma_{5}}{2}
\end{array}\right) \Psi=\left(\begin{array}{l}
\psi_{-R} \\
\psi_{-L}
\end{array}\right),
\end{aligned}
$$

respectively. Here, the subscript \pm and $L(R)$ stand for the chiralities in six and four dimensions, respectively. Using Weyl fermions $\xi_{ \pm}$and $\eta_{ \pm}^{*}$ in four dimensions, $\Psi$ and $\psi_{ \pm L(R)}$ are expressed as

$$
\begin{aligned}
\Psi & =\left(\begin{array}{c}
\xi_{+} \\
\eta_{-}^{*} \\
\xi_{-} \\
\eta_{+}^{*}
\end{array}\right), \quad \psi_{+L}=\left(\begin{array}{c}
\xi_{+} \\
0
\end{array}\right), \quad \psi_{+R}=\left(\begin{array}{c}
0 \\
\eta_{+}^{*}
\end{array}\right), \\
\psi_{-L} & =\left(\begin{array}{c}
\xi_{-} \\
0
\end{array}\right), \quad \psi_{-R}=\left(\begin{array}{c}
0 \\
\eta_{-}^{*}
\end{array}\right) .
\end{aligned}
$$

The charge conjugation of $\Psi$ is defined as

$$
\Psi^{c} \equiv B \Psi^{*},
$$

where $B$ is a $8 \times 8$ matrix which satisfies the relation

$$
B^{-1} \Gamma^{M} B=-\left(\Gamma^{M}\right)^{*} .
$$

The $B$ is given by

$$
B=-i \Gamma_{7} \Gamma^{2} \Gamma^{5}=\left(\begin{array}{cccc}
0 & 0 & 0 & \sigma^{2} \\
0 & 0 & \sigma^{2} & 0 \\
0 & \sigma^{2} & 0 & 0 \\
\sigma^{2} & 0 & 0 & 0
\end{array}\right)
$$

up to a phase factor and, using it, we derive the charge conjugation of $\xi_{ \pm}$and $\eta_{ \pm}^{*}$,

$$
B\left(\begin{array}{c}
\xi_{+} \\
0 \\
0 \\
0
\end{array}\right)=\left(\begin{array}{c}
0 \\
0 \\
0 \\
\sigma^{2} \xi_{+}^{*}
\end{array}\right), \quad B\left(\begin{array}{c}
0 \\
0 \\
0 \\
\eta_{+}^{*}
\end{array}\right)=\left(\begin{array}{c}
\sigma^{2} \eta_{+} \\
0 \\
0 \\
0
\end{array}\right)
$$

and

$$
B\left(\begin{array}{c}
0 \\
0 \\
\xi_{-} \\
0
\end{array}\right)=\left(\begin{array}{c}
0 \\
\sigma^{2} \xi_{-}^{*} \\
0 \\
0
\end{array}\right), \quad B\left(\begin{array}{c}
0 \\
\eta_{-}^{*} \\
0 \\
0
\end{array}\right)=\left(\begin{array}{c}
0 \\
0 \\
\sigma^{2} \eta_{-} \\
0
\end{array}\right) .
$$

From (B12) and (B13), we find that the chirality in six dimensions does not flip under the charge conjugation.

In terms of $\psi_{ \pm L(R)}$, the kinetic terms for $\Psi_{+}$and $\Psi_{-}$are rewritten as

$$
\begin{aligned}
i \bar{\Psi}_{+} \Gamma^{M} D_{M} \Psi_{+}= & i \bar{\Psi}_{+} \Gamma^{\mu} D_{\mu} \Psi_{+}+i \bar{\Psi}_{+} \Gamma^{z} D_{z} \Psi_{+} \\
& +i \bar{\Psi}_{+} \Gamma^{\bar{z}} D_{\bar{z}} \Psi_{+} \\
= & i \bar{\psi}_{+L} \gamma^{\mu} D_{\mu} \psi_{+L}+i \bar{\psi}_{+R} \gamma^{\mu} D_{\mu} \psi_{+R} \\
& -2 \bar{\psi}_{+L} D_{z} \psi_{+R}+2 \bar{\psi}_{+R} D_{\bar{z}} \psi_{+L},
\end{aligned}
$$




$$
\begin{aligned}
i \bar{\Psi}_{-} \Gamma^{M} D_{M} \Psi_{-}= & i \bar{\Psi}_{-} \Gamma^{\mu} D_{\mu} \Psi_{-}+i \bar{\Psi}_{-} \Gamma^{z} D_{z} \Psi_{-}+i \bar{\Psi}_{-} \Gamma^{\bar{z}} D_{\bar{z}} \Psi_{-} \\
= & i \bar{\psi}_{-R} \gamma^{\mu} D_{\mu} \psi_{-R}+i \bar{\psi}_{-L} \gamma^{\mu} D_{\mu} \psi_{-L} \\
& -2 \bar{\psi}_{-R} D_{z} \psi_{-L}+2 \bar{\psi}_{-L} D_{\bar{z}} \psi_{-R},
\end{aligned}
$$

where $\bar{\Psi}_{+}, \bar{\Psi}_{-}, \Gamma^{z}$, and $\Gamma^{\bar{z}}$ are defined by

$$
\begin{aligned}
& \bar{\Psi}_{+} \equiv \Psi_{+}^{\dagger} \Gamma^{0}=\left(\psi_{+L}^{\dagger} \gamma^{0},-\psi_{+R}^{\dagger} \gamma^{0}\right)=\left(\bar{\psi}_{+L},-\bar{\psi}_{+R}\right), \\
& \bar{\Psi}_{-} \equiv \Psi_{-}^{\dagger} \Gamma^{0}=\left(\psi_{-R}^{\dagger} \gamma^{0},-\psi_{-L}^{\dagger} \gamma^{0}\right)=\left(\bar{\psi}_{-R},-\bar{\psi}_{-L}\right),
\end{aligned}
$$

$$
\Gamma^{z} \equiv \Gamma^{5}+i \Gamma^{6}=2 i I_{4 \times 4} \otimes \sigma_{+}=\left(\begin{array}{cc}
0 & 2 i I_{4 \times 4} \\
0 & 0
\end{array}\right),
$$

$$
\Gamma^{\bar{z}} \equiv \Gamma^{5}-i \Gamma^{6}=2 i I_{4 \times 4} \otimes \sigma_{-}=\left(\begin{array}{cc}
0 & 0 \\
2 i I_{4 \times 4} & 0
\end{array}\right) .
$$

Here, $z \equiv x^{5}+i x^{6}$ and $\bar{z} \equiv x^{5}-i x^{6}$. The Kaluza-Klein masses are generated from the terms including $D_{z}$ and $D_{\bar{z}}$ upon compactification.

The $Z_{m}$ elements are the eigenvalues of the representation matrices $T_{\Psi_{ \pm}}\left[U_{a}, \eta_{a \pm}\right]$ for the $Z_{m}$ transformation $z \rightarrow$ $f_{a}(z)[\underbrace{f_{a} \circ f_{a} \cdot \circ f_{a}}_{m}(z)=z]$, operating $\Psi_{ \pm}(x, z, \bar{z})$ such that
$\Psi_{ \pm}\left(x, f_{a}(z), \overline{f_{a}}(\bar{z})\right)=T_{\Psi_{ \pm}}\left[U_{a}, \eta_{a \pm}\right] \Psi_{ \pm}(x, z, \bar{z})$,

where $U_{a}$ represent the representation matrices for the fundamental representation, $\eta_{a \pm}$ are the intrinsic $Z_{m}$ elements, and the subscripts $L$ and $R$ are omitted on $\eta_{a \pm}$. Let the intrinsic $Z_{m}$ elements of $\psi_{ \pm L(R)}$ be $\eta_{a \pm L(R)}$. Then, the intrinsic $Z_{m}$ elements of $\psi_{ \pm L(R)}^{\dagger}$ are $\overline{\eta_{a \pm L(R)}}$ [complex conjugations of $\left.\eta_{a \pm L(R)}\right]$. From the $Z_{m}$ invariance of the kinetic term (B14) and (B15) and the $Z_{m}$ transformation property of the covariant derivative $D_{z} \rightarrow \bar{\rho} D_{z}$ and $D_{\bar{z}} \rightarrow$ $\rho D_{\bar{z}}$ under $z \rightarrow \rho z$ and $\bar{z} \rightarrow \bar{\rho} \bar{z}\left(\rho=e^{2 \pi i / m}, \bar{\rho}=e^{-2 \pi i / m}\right)$, the following relations are derived:

$$
\eta_{a+R}=\rho \eta_{a+L}, \quad \eta_{a-R}=\bar{\rho} \eta_{a-L} .
$$

\section{APPENDIX C: FLAVOR NUMBERS AND CHARGE CONJUGATION}

We give formulas for flavor numbers from a fermion in $\overline{[N, k]}(=[N, N-k])$ and study the relationship between flavor numbers from a fermion in $[N, k]$ and those from a fermion in $[N, k]$ from the viewpoint of charge conjugation.

Under the representation matrices $U_{a}$ with $p_{1}=3$ and $p_{2}=2,[N, N-k]$ is decomposed as

$$
[N, N-k]=\sum_{l_{1}=0}^{N-k} \sum_{l_{2}=0}^{N-k-l_{1}} \sum_{l_{3}=0}^{N-k-l_{1}-l_{2}} \cdots \sum_{l_{n-1}=0}^{N-k-l_{1}-\cdots-l_{n-2}}\left({ }_{3} C_{l_{1}},{ }_{2} C_{l_{2}}, p_{3} C_{l_{3}}, \ldots, p_{n} C_{l_{n}}\right) \text {, }
$$

where $\sum_{i=1}^{n} l_{i}=N-k$. From $[N, N-k]=\overline{[N, k]}$, hereafter we use the decomposition of $\overline{[N, k]}$ such that

$$
\overline{[N, k]}=\sum_{l_{1}=0}^{k} \sum_{l_{2}=0}^{k-l_{1}} \sum_{l_{3}=0}^{k-l_{1}-l_{2}} \cdots \sum_{l_{n-1}=0}^{k-l_{1}-\cdots-l_{n-2}}\left(\overline{{ }_{3} C_{l_{1}}}, \overline{{ }_{2} C_{l_{2}}}, \overline{p_{3} C_{l_{3}}}, \ldots, \overline{p_{n} C_{l_{n}}}\right),
$$

where $\sum_{i=1}^{n} l_{i}=k$. Using the survival hypothesis and the equivalence on charge conjugation in four dimensions, we define the flavor number of each chiral fermion as

$$
\begin{aligned}
& n_{\bar{d}} \equiv\left(\sharp\left(\overline{{ }_{3} C_{2}}, \overline{C_{2} C_{2}}\right)_{R}-\sharp\left(\overline{{ }_{3} C_{1}}, \overline{C_{2} C_{0}}\right)_{R}\right)-\left(\sharp\left(\overline{{ }_{3} C_{2}}, \overline{2} C_{2}\right)_{L}-\sharp\left(\overline{{ }_{3} C_{1}}, \overline{C_{2} C_{0}}\right)_{L}\right), \\
& n_{l} \equiv\left(\sharp\left(\overline{{ }_{3} C_{3}}, \overline{C_{2} C_{1}}\right)_{R}-\sharp\left(\overline{{ }_{3} C_{0}}, \overline{C_{2} C_{1}}\right)_{R}\right)-\left(\sharp\left(\overline{{ }_{3} C_{3}}, \overline{C_{2} C_{1}}\right)_{L}-\sharp\left(\overline{{ }_{3} C_{0}}, \overline{{ }_{2} C_{1}}\right)_{L}\right), \\
& n_{\bar{u}} \equiv\left(\sharp\left(\overline{{ }_{3} C_{2}}, \overline{C_{2} C_{0}}\right)_{R}-\sharp\left(\overline{{ }_{3} C_{1}}, \overline{C_{2} C_{2}}\right)_{R}\right)-\left(\sharp\left(\overline{{ }_{3} C_{2}}, \overline{C_{2} C_{0}}\right)_{L}-\sharp\left(\overline{{ }_{3} C_{1}}, \overline{{ }_{2} C_{2}}\right)_{L}\right), \\
& n_{\bar{e}} \equiv\left(\sharp\left(\overline{{ }_{3} C_{0}}, \overline{C_{2} C_{2}}\right)_{R}-\sharp\left(\overline{{ }_{3} C_{3}}, \overline{C_{2} C_{0}}\right)_{R}\right)-\left(\sharp\left(\overline{{ }_{3} C_{0}}, \overline{C_{2} C_{2}}\right)_{L}-\sharp\left(\overline{{ }_{3} C_{3}}, \overline{C_{2} C_{0}}\right)_{L}\right), \\
& n_{q} \equiv\left(\sharp\left(\overline{{ }_{3} C_{1}}, \overline{C_{2} C_{1}}\right)_{R}-\sharp\left(\overline{{ }_{3} C_{2}}, \overline{C_{2} C_{1}}\right)_{R}\right)-\left(\sharp\left(\overline{{ }_{3} C_{1}}, \overline{C_{2} C_{1}}\right)_{L}-\sharp\left(\overline{{ }_{3} C_{2}}, \overline{{ }_{2} C_{1}}\right)_{L}\right),
\end{aligned}
$$

where $\sharp$ represents the number of zero modes for each multiplet. The total number of neutrino singlets $\left(\nu_{R}\right)^{c}$ and/or $\nu_{R}$ is defined as

$$
n_{\bar{\nu}} \equiv \sharp\left(\overline{C_{3} C_{0}},{ }_{2} C_{0}\right)_{R}+\sharp\left(\overline{{ }_{3} C_{3}}, \overline{C_{2} C_{2}}\right)_{R}+\sharp\left(\overline{{ }_{3} C_{0}},{ }_{2} \bar{C}_{0}\right)_{L}+\sharp\left(\overline{{ }_{3} C_{3}}, \overline{C_{2}}\right)_{L} .
$$


Note that we have relations

$$
\left(\overline{{ }_{3} C_{l_{1}}}, \overline{{ }_{2} C_{l_{2}}}\right)_{R(L)}=\left({ }_{3} C_{3-l_{1}},{ }_{2} C_{2-l_{2}}\right)_{R(L)} .
$$

Formulas for the SM species and neutrino singlets derived from a pair of six-dimensional Weyl fermions $\left(\Psi_{+}, \Psi_{-}\right)$in $\overline{[N, k]}$ are given by

$$
\begin{aligned}
& \left.n_{\bar{d}}\right|_{[\overline{[N, k]}}=\sum_{ \pm} \sum_{\left(l_{1}, l_{2}\right)=(2,2),(1,0)} \sum_{l_{3}=0}^{k-l_{1}-l_{2}} \cdots \sum_{l_{n-1}=0}^{k-l_{1}-\cdots-l_{n-2}}(-1)^{l_{1}+l_{2}} \tilde{P}_{m k \pm p_{3}} C_{l_{3}} \cdots{ }_{p_{n}} C_{l_{n}}, \\
& \left.n_{l}\right|_{[N, k]}=\sum_{ \pm} \sum_{\left(l_{1}, l_{2}\right)=(3,1),(0,1)} \sum_{l_{3}=0}^{k-l_{1}-l_{2}} \cdots \sum_{l_{n-1}=0}^{k-l_{1}-\cdots-l_{n-2}}(-1)^{l_{1}+l_{2}} \tilde{P}_{m k \pm p_{3}} C_{l_{3}} \cdots_{p_{n}} C_{l_{n}}, \\
& \left.n_{\bar{u}}\right|_{[N, k]}=\sum_{ \pm} \sum_{\left(l_{1}, l_{2}\right)=(2,0),(1,2)} \sum_{l_{3}=0}^{k-l_{1}-l_{2}} \cdots \sum_{l_{n-1}=0}^{k-l_{1}-\cdots-l_{n-2}}(-1)^{l_{1}+l_{2}} \tilde{P}_{m k \pm p_{3}} C_{l_{3}} \cdots_{p_{n}} C_{l_{n}}, \\
& \left.n_{\bar{e}}\right|_{[N, k]}=\sum_{ \pm} \sum_{\left(l_{1}, l_{2}\right)=(0,2),(3,0)} \sum_{l_{3}=0}^{k-l_{1}-l_{2}} \cdots \sum_{l_{n-1}=0}^{k-l_{1}-\cdots-l_{n-2}}(-1)^{l_{1}+l_{2}} \tilde{P}_{m k \pm p_{3}} C_{l_{3}} \cdots_{p_{n}} C_{l_{n}}, \\
& \left.n_{q}\right|_{[N, k]}=\sum_{ \pm} \sum_{\left(l_{1}, l_{2}\right)=(1,1),(2,1)} \sum_{l_{3}=0}^{k-l_{1}-l_{2}} \cdots \sum_{l_{n-1}=0}^{k-l_{1}-\cdots-l_{n-2}}(-1)^{l_{1}+l_{2}} \tilde{P}_{m k \pm p_{3}} C_{l_{3}} \cdots{ }_{p_{n}} C_{l_{n}}, \\
& \left.n_{\bar{\nu}}\right|_{[\overline{[N, k]}}=\sum_{ \pm} \sum_{\left(l_{1}, l_{2}\right)=(0,0),(3,2)} \sum_{l_{3}=0}^{k-l_{1}-l_{2}} \cdots \sum_{l_{n-1}=0}^{k-l_{1}-\cdots-l_{n-2}} \tilde{P}_{m k p_{3}}^{(\nu)} C_{l_{3}} \cdots{ }_{p_{n}} C_{l_{n}},
\end{aligned}
$$

where $\tilde{P}_{m k \pm}$ and $\tilde{P}_{m k \pm}^{(\nu)}$ are defined by

$$
\tilde{P}_{m k \pm} \equiv \tilde{P}_{m k \pm R}-\tilde{P}_{m k \pm L}, \quad \tilde{P}_{m k \pm}^{(\nu)} \equiv \tilde{P}_{m k \pm R}+\tilde{P}_{m k \pm L}
$$

respectively. The $\tilde{P}_{m k \pm R(L)}$ are projection operators to pick out zero modes of $\psi_{ \pm R(L)}$ in $\overline{[N, k]}$, and they are listed in Table IX.

In Table IX, each operator is defined by

$$
\begin{aligned}
& \tilde{P}_{2 k \pm}^{\left((-1)^{n_{0}},(-1)^{n_{1}},(-1)^{n_{2}}\right)} \equiv \frac{1}{8}\left\{1+(-1)^{n_{0}} \tilde{\mathcal{P}}_{0 \pm}^{(k)}\right\}\left\{1+(-1)^{n_{1}} \tilde{\mathcal{P}}_{1 \pm}^{(k)}\right\}\left\{1+(-1)^{n_{2}} \tilde{\mathcal{P}}_{2 \pm}^{(k)}\right\}, \\
& \tilde{P}_{3 k \pm}^{\left(\omega^{n_{0}}, \omega^{n_{1}}\right)} \equiv \frac{1}{9}\left\{1+\bar{\omega}^{n_{0}} \tilde{\mathcal{P}}_{0 \pm}^{(k)}+\bar{\omega}^{2 n_{0}}\left(\tilde{\mathcal{P}}_{0 \pm}^{(k)}\right)^{2}\right\}\left\{1+\bar{\omega}^{n_{1}} \tilde{\mathcal{P}}_{1 \pm}^{(k)}+\bar{\omega}^{2 n_{1}}\left(\tilde{\mathcal{P}}_{1 \pm}^{(k)}\right)^{2}\right\},
\end{aligned}
$$

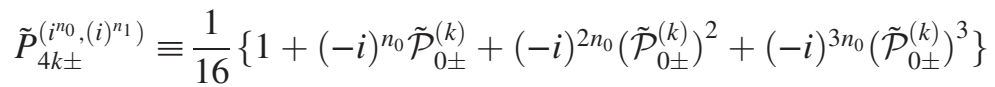

$$
\begin{aligned}
& \times\left\{1+(-i)^{n_{1}} \tilde{\mathcal{P}}_{1 \pm}^{(k)}+(-i)^{2 n_{1}}\left(\tilde{\mathcal{P}}_{1 \pm}^{(k)}\right)^{2}+(-i)^{3 n_{1}}\left(\tilde{\mathcal{P}}_{1 \pm}^{(k)}\right)^{3}\right\}, \\
& \tilde{P}_{6 k \pm}^{\left(\varphi^{n_{0}}\right)} \equiv \frac{1}{6}\left\{1+\bar{\varphi}^{n_{0}} \tilde{\mathcal{P}}_{0 \pm}^{(k)}+\bar{\varphi}^{2 n_{0}}\left(\tilde{\mathcal{P}}_{0 \pm}^{(k)}\right)^{2}+\bar{\varphi}^{3 n_{0}}\left(\tilde{\mathcal{P}}_{0 \pm}^{(k)}\right)^{3}+\bar{\varphi}^{4 n_{0}}\left(\tilde{\mathcal{P}}_{0 \pm}^{(k)}\right)^{4}+\bar{\varphi}^{5 n_{0}}\left(\tilde{\mathcal{P}}_{0 \pm}^{(k)}\right)^{5}\right\},
\end{aligned}
$$

where $\tilde{\mathcal{P}}_{a \pm}^{(k)}$ are the $Z_{m}$ elements. For instance, $\tilde{P}_{3 k \pm}^{\left(\omega^{n_{0}}, \omega^{n_{1}}\right)}$ is a projection operator to pick out modes with $\tilde{\mathcal{P}}_{0 \pm}^{(k)}=\omega^{n_{0}}$ and $\tilde{\mathcal{P}}_{1 \pm}^{(k)}=\omega^{n_{1}}$ in $\Psi_{ \pm}$. By the insertion of $(-1)^{l_{1}+l_{2}}$, we obtain $\sharp\left({ }_{3} C_{l_{1}},{ }_{2} C_{l_{2}}\right)_{R(L)}$ for $l_{1}+l_{2}=$ even integer and $-\sharp\left(\overline{{ }_{3} C_{l_{1}}}, \overline{{ }_{2} C_{l_{2}}}\right)_{R(L)}$ for $l_{1}+l_{2}=$ odd integer. 
TABLE IX. The projection operators $\tilde{P}_{m k \pm R(L)}$.

\begin{tabular}{lcccc}
\hline \hline$T^{2} / Z_{m}$ & $\tilde{P}_{m k+R}$ & $\tilde{P}_{m k+L}$ & $\tilde{P}_{m k-R}$ & $\tilde{P}_{m k-L}$ \\
\hline$T^{2} / Z_{2}$ & $\tilde{P}_{2 k+}^{(1,1,1)}$ & $\tilde{P}_{2 k+}^{(-1,-1,-1)}$ & $\tilde{P}_{2 k-}^{(1,1,1)}$ & $\tilde{P}_{2 k-}^{(-1,-1,-1)}$ \\
$T^{2} / Z_{3}$ & $\tilde{P}_{3 k+}^{(1,1)}$ & $\tilde{P}_{3 k+}^{(\omega, \omega)}$ & $\tilde{P}_{3 k-}^{(1,1)}$ & $\tilde{P}_{3 k-}^{(\bar{\omega}, \bar{\omega})}$ \\
$T^{2} / Z_{4}$ & $\tilde{P}_{4 k+}^{(1,1)}$ & $\tilde{P}_{4 k+}^{(i, i)}$ & $\tilde{P}_{4 k-}^{(1,1)}$ & $\tilde{P}_{4 k-}^{(-i,-i)}$ \\
$T^{2} / Z_{6}$ & $\tilde{P}_{6 k+}^{(1)}$ & $\tilde{P}_{6 k+}^{(\varphi)}$ & $\tilde{P}_{6 k-}^{(1)}$ & $\tilde{P}_{6 k-}^{(\bar{\varphi})}$ \\
\hline \hline
\end{tabular}

The $\tilde{\mathcal{P}}_{a \pm}^{(k)}$ of $\left(\overline{{ }_{3} C_{l_{1}}}, \overline{{ }_{2} C_{l_{2}}}, \ldots, \overline{p_{n} C_{l_{n}}}\right)$ are given by

$$
\begin{aligned}
& \tilde{\mathcal{P}}_{0 \pm}^{(k)}=(-1)^{l_{1}+l_{2}+l_{3}+l_{4}-k} \tilde{\eta}_{0 \pm}^{(k)}, \\
& \tilde{\mathcal{P}}_{1 \pm}^{(k)}=(-1)^{l_{1}+l_{2}+l_{5}+l_{6}-k} \tilde{\eta}_{1 \pm}^{(k)}, \\
& \tilde{\mathcal{P}}_{2 \pm}^{(k)}=(-1)^{l_{1}+l_{3}+l_{5}+l_{7}-k} \tilde{\eta}_{2 \pm}^{(k)}
\end{aligned}
$$

for (3.22);

$$
\begin{aligned}
& \tilde{\mathcal{P}}_{0 \pm}^{(k)}=\bar{\omega}^{l_{1}+l_{2}+l_{3}+2\left(l_{4}+l_{5}+l_{6}\right)-k} \tilde{\eta}_{0 \pm}^{(k)}, \\
& \tilde{\mathcal{P}}_{1 \pm}^{(k)}=\bar{\omega}^{l_{1}+l_{4}+l_{7}+2\left(l_{2}+l_{5}+l_{8}\right)-k} \tilde{\eta}_{1 \pm}^{(k)}
\end{aligned}
$$

for (3.24);

$$
\begin{aligned}
& \tilde{\mathcal{P}}_{0 \pm}^{(k)}=(-i)^{l_{1}+l_{2}+2\left(l_{3}+l_{4}\right)+3\left(l_{5}+l_{6}\right)-k} \tilde{\eta}_{0 \pm}^{(k)}, \\
& \tilde{\mathcal{P}}_{1 \pm}^{(k)}=(-i)^{l_{1}+l_{6}+2\left(l_{4}+l_{7}\right)+3\left(l_{2}+l_{5}\right)-k} \tilde{\eta}_{1 \pm}^{(k)}
\end{aligned}
$$

for (3.26); and

$$
\tilde{\mathcal{P}}_{0 \pm}^{(k)}=\bar{\varphi}^{l_{1}+2 l_{2}+3 l_{3}+4 l_{4}+5 l_{5}-k} \tilde{\eta}_{0 \pm}^{(k)}
$$

for (3.28). The subscripts $L$ and $R$ on the intrinsic $Z_{m}$ elements are omitted in (C21)-(C24). Notice that complex values $\omega, i$, and $\varphi$ in (3.25), (3.27), and (3.29) are replaced into their complex conjugated ones, respectively, in (C22)(C24), because $U_{a}^{*}$ operate fields multiple times in place of $U_{a}$.

From (2.6), $\tilde{\eta}_{a \pm L}^{(k)}$ are determined from $\tilde{\eta}_{a \pm R}^{(k)}$ as

$$
\tilde{\eta}_{a+L}^{(k)}=\bar{\rho} \tilde{\eta}_{a+R}^{(k)}, \quad \tilde{\eta}_{a-L}^{(k)}=\rho \tilde{\eta}_{a-R}^{(k)} .
$$

In the case that $\tilde{\eta}_{a \pm R}^{(k)}=\overline{\eta_{a \pm L}^{(k)}}$, we have the relations

$$
\tilde{\mathcal{P}}_{a \pm}^{(k)}=\overline{\mathcal{P}_{a \pm}^{(k)}}
$$

and derive the relations

$$
\begin{aligned}
& \tilde{P}_{2 k \pm}^{\left((-1)^{n_{0}},(-1)^{n_{1}},(-1)^{n_{2}}\right)}=P_{2 k \pm}^{\left((-1)^{n_{0}},(-1)^{n_{1}},(-1)^{n_{2}}\right)}, \\
& \tilde{P}_{m k \pm}^{\left(\rho^{n_{0}}, \rho^{n_{1}}\right)}=\overline{P_{m k \pm}^{\left(\bar{\rho}^{n_{0}}, \bar{\rho}^{n_{1}}\right)}}=P_{m k \pm}^{\left(\bar{\rho}^{n_{0}}, \bar{\rho}^{n_{1}}\right)}(m=3,4), \\
& \tilde{P}_{6 k \pm}^{\left(\varphi^{n_{0}}\right)}=\overline{P_{6 k \pm}^{\left(\bar{\varphi}^{n_{0}}\right)}}=P_{6 k \pm}^{\left(\bar{\varphi}^{n_{0}}\right)} .
\end{aligned}
$$

In the last equality in the above second relation, we use the fact that the projection operators take a real number 1 or 0 . From $(\mathrm{C} 27)$, we find that the flavor numbers derived from the projection by $(-1)^{l_{1}+l_{2}} \tilde{P}_{m k \pm}$ are equal to those from that by $(-1)^{l_{1}+l_{2}} \overline{P_{m k \pm}}=(-1)^{l_{1}+l_{2}} P_{m k \pm}$. In this way, we have a feature that each flavor number from a fermion in $[N, k]$ with intrinsic $Z_{m}$ elements $\eta_{a \pm}^{(k)}$ is equal to that from a fermion in $\overline{[N, k]}(=[N, N-k])$ with those satisfying $\tilde{\eta}_{a \pm R}^{(k)}=\overline{\eta_{a \pm L}^{(k)}}\left[\right.$ suitable $\left.\eta_{a \pm}^{(N-k)}\right]$. In other words, there is a one-to-one correspondence between zero modes from a Weyl fermion in $[N, k]$ with $\eta_{a \pm}^{(k)}$ and those from a Weyl fermion in $[N, N-k]$ with suitable $\eta_{a \pm}^{(N-k)}$.

Finally, let us obtain suitable $\eta_{a \pm}^{(N-k)}$ to hold the abovestated correspondence, in the case with (3.24) of $T^{2} / Z_{3}$. In this case, $\mathcal{P}_{a \pm}^{(N-k)}$ are given by

$\mathcal{P}_{0 \pm}^{(N-k)}=\omega^{l_{1}+l_{2}+l_{3}+2\left(l_{4}+l_{5}+l_{6}\right)-(N-k)} \eta_{0 \pm}^{(N-k)}$,

$\mathcal{P}_{1 \pm}^{(N-k)}=\omega^{l_{1}+l_{4}+l_{7}+2\left(l_{2}+l_{5}+l_{8}\right)-(N-k)} \eta_{1 \pm}^{(N-k)}$.

By replacing $l_{i}$ into $p_{i}-l_{i}$ in $\mathcal{P}_{0 \pm}^{(N-k)}$ and $\mathcal{P}_{1 \pm}^{(N-k)}$, we obtain $\tilde{\mathcal{P}}_{0 \pm}^{(k)}$ and $\tilde{\mathcal{P}}_{1 \pm}^{(k)}$ such that

$\tilde{\mathcal{P}}_{0 \pm}^{(k)}=\bar{\omega}^{l_{1}+l_{2}+l_{3}+2\left(l_{4}+l_{5}+l_{6}\right)-k} \omega^{p_{1}+p_{2}+p_{3}+2\left(p_{4}+p_{5}+p_{6}\right)-N} \eta_{0 \pm}^{(N-k)}$,

$\tilde{\mathcal{P}}_{1 \pm}^{(k)}=\bar{\omega}^{l_{1}+l_{4}+l_{7}+2\left(l_{2}+l_{5}+l_{8}\right)-k} \omega^{p_{1}+p_{4}+p_{7}+2\left(p_{2}+p_{5}+p_{8}\right)-N} \eta_{1 \pm}^{(N-k)}$.

Using (C22), (C29), (C30), and $\tilde{\eta}_{a \pm R}^{(k)}=\overline{\eta_{a \pm L}^{(k)}}$, we derive the relations

$$
\tilde{\eta}_{0 \pm R}^{(k)}=\omega^{p_{1}+p_{2}+p_{3}+2\left(p_{4}+p_{5}+p_{6}\right)-N} \eta_{0 \pm R}^{(N-k)}=\overline{\eta_{0 \pm L}^{(k)}},
$$

$$
\tilde{\eta}_{1 \pm R}^{(k)}=\omega^{p_{1}+p_{4}+p_{7}+2\left(p_{2}+p_{5}+p_{8}\right)-N} \eta_{1 \pm R}^{(N-k)}=\overline{\eta_{1 \pm L}^{(k)}} .
$$

The equivalence based on the relations (C31) and (C32) is illustrated with the particle contents listed in Tables VII and VIII. 
[1] P. Ramond, arXiv:hep-ph/9809459.

[2] M. Gell-Mann, P. Ramond, and R. Slansky, in Supergravity, edited by $P$. van Nieuwenhuizen and D.Z. Freedman (North Holland, Amsterdam, 1979).

[3] H. Georgi, Nucl. Phys. B156, 126 (1979).

[4] P. H. Frampton, Phys. Lett. 88B, 299 (1979).

[5] P. H. Frampton, Phys. Lett. 89B, 352 (1980).

[6] F. Wilczek and A. Zee, Phys. Rev. D 25, 553 (1982).

[7] T. Kugo and T. Yanagida, Phys. Lett. 134B, 313 (1984).

[8] R. Barbieri, D. V. Nanopoulos, G. Morchio, and F. Strocchi, Phys. Lett. 90B, 91 (1980).

[9] P. Candelas, G. T. Horowitz, A. Strominger, and E. Witten, Nucl. Phys. B258, 46 (1985).

[10] L. Dixon, J. A. Harvey, C. Vafa, and E. Witten, Nucl. Phys. B261, 678 (1985).

[11] L. Dixon, J. A. Harvey, C. Vafa, and E. Witten, Nucl. Phys. B274, 285 (1986).

[12] Y. Kawamura, Prog. Theor. Phys. 105, 999 (2001).

[13] L. Hall and Y. Nomura, Phys. Rev. D 64, 055003 (2001).

[14] K. S. Babu, S. M. Barr, and B. Kyae, Phys. Rev. D 65, 115008 (2002).

[15] T. Watari and T. Yanagida, Phys. Lett. B 532, 252 (2002).

[16] I. Gogoladze, Y. Mimura, and S. Nandi, Phys. Lett. B 562, 307 (2003).

[17] I. Gogoladze, T. Li, Y. Mimura, and S. Nandi, Phys. Rev. D 72, 055006 (2005).

[18] I. Gogoladze, C. A. Lee, Y. Mimura, and Q. Shafi, Phys. Lett. B 649, 212 (2007).

[19] I. Gogoladze, Y. Mimura, and S. Nandi, Phys. Rev. Lett. 91, 141801 (2003).

[20] Y. Kawamura, T. Kinami, and K. Oda, Phys. Rev. D 76, 035001 (2007).

[21] Y. Kawamura and T. Miura, Phys. Rev. D 81, 075011 (2010).

[22] Y. Fujimoto, T. Kobayashi, T. Miura, K. Nishiwaki, and M. Sakamoto, Phys. Rev. D 87, 086001 (2013).

[23] Y. Goto, Y. Kawamura, and T. Miura, Phys. Rev. D 88, 055016 (2013).

[24] N. Yamatsu, Prog. Theor. Exp. Phys. 2017, 091B01 (2017).

[25] N. Yamatsu, Prog. Theor. Exp. Phys. 2017, 101B01 (2017).

[26] Y. Goto, Y. Kawamura, and T. Miura, Int. J. Mod. Phys. A29, 1450150 (2014).

[27] Y. Goto and Y. Kawamura, Phys. Lett. B 752, 252 (2016).

[28] T. Yamashita (private communication).

[29] Y. Kawamura, T. Kinami, and T. Miura, Prog. Theor. Phys. 120, 815 (2008).

[30] Y. Kawamura and T. Miura, Prog. Theor. Phys. 122, 847 (2009).

[31] Y. Hosotani, Phys. Lett. 126B, 309 (1983).

[32] Y. Hosotani, Ann. Phys. (N.Y.) 190, 233 (1989).

[33] N. Haba, M. Harada, Y. Hosotani, and Y. Kawamura, Nucl. Phys. B657, 169 (2003); B669, 381(E) (2003).
[34] N. Haba, Y. Hosotani, and Y. Kawamura, Prog. Theor. Phys. 111, 265 (2004).

[35] L. Antoniadis, Phys. Lett. B 246, 377 (1990).

[36] P. Hořava and E. Witten, Nucl. Phys. B460, 506 (1996).

[37] P. Hořava and E. Witten, Nucl. Phys. B475, 94 (1996).

[38] E. A. Mirabelli and M. Peskin, Phys. Rev. D 58, 065002 (1998).

[39] A. Pomarol and M. Quriós, Phys. Lett. B 438, 255 (1998).

[40] J. Scherk and J. H. Schwarz, Phys. Lett. 82B, 60 (1979).

[41] J. Scherk and J. H. Schwarz, Nucl. Phys. B153, 61 (1979).

[42] Y. Kawamura, Prog. Theor. Phys. 103, 613 (2000).

[43] B. A. Dobrescu and E. Poppitz, Phys. Rev. Lett. 87, 031801 (2001).

[44] N. Borghini, Y. Gouvemeur, and M. H. G. Tytgat, Phys. Rev. D 65, 025017 (2001).

[45] P. Minkowski, Phys. Lett. 67B, 421 (1977).

[46] T. Yanagida, in Proceedings of Workshop on the Unified Theory and the Baryon Number in the Universe, edited by O. Sawada and A. Sugamoto (KEK Report No. 79-18, 1979).

[47] Y. Goto and Y. Kawamura (to be published).

[48] M. Drees, Phys. Lett. B 181, 279 (1986).

[49] J. S. Hagelin and S. Kelley, Nucl. Phys. B342, 95 (1990).

[50] Y. Kawamura, H. Murayama, and M. Yamaguchi, Phys. Lett. B 324, 52 (1994).

[51] Y. Kawamura, H. Murayama, and M. Yamaguchi, Phys. Rev. D 51, 1337 (1995).

[52] Y. Kawamura and M. Tanaka, Prog. Theor. Phys. 91, 949 (1994).

[53] Y. Kawamura and T. Kinami, Int. J. Mod. Phys. A22, 4617 (2007).

[54] Y. Kawamura and T. Kinami, Prog. Theor. Phys. 119, 285 (2008).

[55] Y. Kawamura, T. Kinami, and T. Miura, J. High Energy Phys. 01 (2009) 064.

[56] C. D. Froggatt and H. B. Nielsen, Nucl. Phys. B147, 277 (1979).

[57] K. Yoshioka, Mod. Phys. Lett. A 15, 29 (2000).

[58] L. E. Ibáñez and A. M. Uranga, String Theory and Particle Physics (Cambridge University Press, Cambridge, 2012).

[59] S. Mizoguchi and M. Yata, Prog. Theor. Exp. Phys. 2013, 053B01 (2013).

[60] S. Mizoguchi, J. High Energy Phys. 07 (2014) 018.

[61] N. M. Borštnik and H. B. Nielsen, J. Math. Phys. (N.Y.) 44, 4817 (2003).

[62] A. B. Bračič and N. M. Borštnik, Phys. Rev. D 74, 073013 (2006).

[63] M. Y. Khlopov, Cosmoparticle Physics (World Scientific, Singapore, 1999).

[64] Y. Kawamura and T. Miura, Int. J. Mod. Phys. A28, 1350055 (2013). 